\title{
Functional characterization of EZH $2 \beta$ reveals the increased complexity of EZH2 isoforms involved in the regulation of mammalian gene expression
}

Adrienne Grzenda', Gwen Lomberk', Phyllis Svingen', Angela Mathison', Ezequiel Calvo², Juan lovanna ${ }^{3}$, Yuning Xiong ${ }^{1}$, William Faubion ${ }^{1}$ and Raul Urrutia ${ }^{1,3,4,5^{*}}$

\begin{abstract}
Background: Histone methyltransferase enhancer of zeste homologue 2 (EZH2) forms an obligate repressive complex with suppressor of zeste 12 and embryonic ectoderm development, which is thought, along with EZH1, to be primarily responsible for mediating Polycomb-dependent gene silencing. Polycomb-mediated repression influences gene expression across the entire gamut of biological processes, including development, differentiation and cellular proliferation. Deregulation of EZH2 expression is implicated in numerous complex human diseases. To date, most EZH2-mediated function has been primarily ascribed to a single protein product of the EZH2 locus.

Results: We report that the EZH2 locus undergoes alternative splicing to yield at least two structurally and functionally distinct EZH2 methyltransferases. The longest protein encoded by this locus is the conventional enzyme, which we refer to as $\mathrm{EZH} 2 \mathrm{a}$, whereas $\mathrm{EZH} 2 \beta$, characterized here, represents a novel isoform. We find that $\mathrm{EZH} 2 \beta$ localizes to the cell nucleus, complexes with embryonic ectoderm development and suppressor of zeste 12, trimethylates histone 3 at lysine 27, and mediates silencing of target promoters. At the cell biological level, we find that increased $E Z \mathrm{H} 2 \beta$ induces cell proliferation, demonstrating that this protein is functional in the regulation of processes previously attributed to EZH2a. Biochemically, through the use of genome-wide expression profiling, we demonstrate that $\mathrm{EZ} 2 \mathrm{H} \beta$ governs a pattern of gene repression that is often ontologically redundant from that of EZH2a, but also divergent for a wide variety of specific target genes.

Conclusions: Combined, these results demonstrate that an expanded repertoire of EZH 2 writers can modulate histone code instruction during histone 3 lysine 27-mediated gene silencing. These data support the notion that the regulation of EZH2-mediated gene silencing is more complex than previously anticipated and should guide the design and interpretation of future studies aimed at understanding the biochemical and biological roles of this important family of epigenomic regulators.
\end{abstract}

Keywords: Chromatin, Enhancer of zeste homologue 2, Epigenetics, EZH2, Histone methyltransferase, Polycomb, Polycomb repressive complex 2, PRC2

\footnotetext{
* Correspondence: urrutia.raul@mayo.edu

'Laboratory of Epigenetics and Chromatin Dynamics, Mayo Clinic, Rochester,

MN 55905, USA

${ }^{3}$ INSERM U.624, Stress Cellulaire, 163 Avenue de Luminy, Case 915, Parc

Scientifique et Technologique de Luminy, 13288, Marseille Cedex 9, France

Full list of author information is available at the end of the article
} 


\section{Background}

The currently accepted hierarchical model of Polycombmediated gene repression begins with the trimethylation of histone 3 at lysine 27 (H3-K27me3) through the action of Polycomb repressive complex (PRC) 2, a multisubunit complex. The H3-K27me3 mark subsequently recruits $\mathrm{PRC1}$, leading to the propagation of the repressed state through a variety of mechanisms, including chromatin compaction and recruitment of other chromatinremodeling enzymes, such as DNA methyltransferases [1]. However, it must be noted that alternative pathways for PRC1 recruitment, independent of inscription of the H3-K27me3 mark, have also been observed [2]. The enzymatic engine of PRC2 is enhancer of zeste homologue 2 (EZH2), which possesses the evolutionarily conserved SET domain that confers the complex with its histone methyltransferase (HMT) activity [3,4]. The catalytic function of EZH2 is strictly dependent on the presence of both WD40-repeat protein embryonic ectoderm development (EED) and zinc finger protein suppressor of zeste 12 (SUZ12), which serve to link and stabilize the enzyme to its histone substrate [5-9]. A number of other proteins, such as retinoblastoma binding proteins 4 and 7 (RBBP4/7), Adipocyte enhancer-binding protein 2 and PHD finger protein 1 enhance the enzymatic function of the complex [5,10-12]. Thus, within these complexes, EZH2 proteins serve as the key histone code writers of the H3-K27me3 mark that leads to long-term epigenetic gene silencing.

PRC2 proteins are extremely well conserved from plants to humans, indicating the fundamental importance of this epigenetic mechanism to organism development and survival [13]. Interestingly, several alternative PRC2 complexes have been identified. EED undergoes alternative translation to yield four protein products. PRC2 complex was defined as a complex between EZH2, SUZ12, RBBP4/7 and the longest isoform of EED, EED1. However, a second complex, PRC3, was found with the substitution of the shortest isoforms of EED, EED3/4, that was capable of H3-K27 methylation [14]. Another alternative complex, PRC4, was found with the substitution of EED2, an isoform normally undetectable in differentiated cells but prevalent in embryonic stem cells and transformed tissues [15]. EZH1, a homologue of EZH2 encoded at a separate locus, is also capable of H3-K27 trimethylation and transcriptional silencing. EZH1 forms a non-canonical PRC2 complex with EED and SUZ12. However, current evidence supports the idea that PRC2-EZH1-mediated H3-K27 trimethylation is less widespread than for PRC2-EZH2. PRC2-EZH1, for instance, has been shown to repress transcription through chromatin compaction in the absence of methyltransferase co-factor S-adenosyl methionine [10]. Together, these data suggest a more intricate regulation of H3-K37me3 deposition than previously anticipated.
The multiplicity of downstream biological functions mediated by EZH2 points to a pervasiveness of Polycombmediated repression well beyond development. Roles for EZH2 have been identified in cell cycle, cellular differentiation and pluripotency, among many others [16]. Pathologically, EZH2 has been implicated in the neoplastic transformation of a number of cell types, including for many solid tumors and hematopoietic malignancies. Levels of EZH2 strongly associate with the severity of malignant progression and poor prognosis in breast and prostate cancer $[17,18]$. The medical relevance of this observation is congruent with the functions for EZH2 as revealed by experimental methods. For instance, EZH2 overexpression promotes cellular proliferation [19-24], migration [25-27], angiogenesis [28] and survival [29,30]. Thus, both basic and translational investigations have established a solid role for EZH2 and its partners as an epigenetic system involved in oncogenesis (epigenetic oncogenes), for which detailed mechanisms underlying their function have become an area of intensive investigation.

The current study increases our knowledge on the complexity of EZH2-mediated processes by providing biochemical evidence revealing extended isoform diversity within EZH2 proteins that function in mammalian cells. Indeed, our molecular and functional data indicate that the $E Z H 2$ locus encodes a novel isoform, EZH2 $\beta$. This isoform localizes to the cell nucleus, complexes with EED and SUZ12, and binds to promoters where it increases $\mathrm{H} 3-\mathrm{K} 27 \mathrm{me} 3$ levels, all properties in common with EZH $2 \alpha$ protein. Importantly, however, EZH2 $\beta$ participates in the regulation of gene expression with a pattern that is not only shared but also distinct from that regulated by $\mathrm{EZH} 2 \alpha$, pointing to both redundancy and specialization within members of this HMT family of proteins. Combined, these results reveal that the regulation of H3-K27 methylation is more complex than previously anticipated and expands our knowledge of how cells generate and use different histone code writers to achieve distinct biochemical and biological functions. This new knowledge must be taken into consideration in the design and interpretation of studies on gene expression, distinct cell functions, single target gene promoters or genome-wide epigenomics, as it reveals for the first time the need for isoform-specific tools to dissect Polycomb functions.

\section{Results}

Identification of $\mathrm{EZH} 2 \beta$ reveals the existence of an expanded repertoire of EZH2 isoforms widely expressed in human tissues

The current study initiated from investigations on the role of the $E Z H 2$ locus in the proliferative response, as previous reports implicated overexpression of this HMT during neoplastic transformation in a variety of cancers 
[31]. Initial western blot analyses in pancreatic cancer cells revealed the presence of multiple EZH2-positive bands (Additional file 1: Figure S1). To date, over 30 different EZH2 mRNAs have been validated by highthroughput genomic sequencing efforts. One of the proteins generated from this locus, EZH $2 \alpha$, encoded by 20 exons, is the HMT classically associated with the function of the PRC2 complex (Figure 1A and Table 1). $\mathrm{EZH} 2 \beta$, a novel isoform that the current study functionally characterizes in better detail, skips exon 4 of EZH $2 \alpha$ and utilizes an alternative $5^{\prime}$ splice donor on EZH $2 \alpha$ exon $8 / \mathrm{EZH} 2 \beta$ exon 7 . At the protein sequence level, EZH $2 \alpha$ and EZH2 $\beta$ differ by 44 amino acids, measuring 751 and 707 amino acids, respectively (Figure 1B). A highly similar third splice variant encoding five less amino acids than EZH $2 \alpha$ has also been cited as EZH2. Structural comparison of these closely related variants does not reveal any noticeable differences that would suggest differing function and thereby have been considered interchangeable in the literature.

For the purposes of our manuscript, we have selected the longest transcriptional variant (751 amino acids) as the reference sequence to provide a numbering framework for our bioinformatics. Compared with this reference EZH $2 \alpha$ sequence, the shorter EZH2 $\beta$ displays conservation of several domains, namely the nuclear localization signals, SANT (DNA-binding domains) and the SET domain. Together, the conservation of these domains should confer this protein with the ability to localize to the nucleus and function as an HMT (Figure 1B). Notably, the regions that have previously been described to interact with the obligate EZH $2 \alpha$ co-factors SUZ12 and EED are also preserved in EZH2 $\beta[7,32]$, suggesting that different EZH2 gene products complex with molecules significant to its major biochemical function. Additionally, comparison of all currently available genome sequences generated by world-wide sequencing efforts revealed that alternative splicing of the $E Z H 2$ locus is conserved from invertebrates to vertebrates. The number of predicted EZH2 orthologs within each of the surveyed species suggests multiple expansion and reduction events may have occurred during the evolution of the protein as evolutionary distance increases from invertebrates to higher-order mammals (Figure $1 \mathrm{C}$ ). We find that the EZH $2 \alpha$ and $\mathrm{EZH} 2 \beta$ are predicted to be greater than $99 \%$ conserved in higher-order mammals (Table 2), suggesting that these two proteins may account for a large number of the functions evolutionarily selected for the $E Z H 2$ locus.

Using a panel of 22 different tissues, we demonstrated that these two transcripts share a similar expression profile in most human organs. Additional comparative analyses of the expression pattern for other Polycombrelated co-factors demonstrated that tissues expressing high levels of $\mathrm{EZH} 2 \alpha$ or $\mathrm{EZH} 2 \beta$ transcripts display a concordant enrichment of transcripts encoding other PRC2 complex proteins, ones previously described to be exclusive partners of the conventional enzyme, EZH $2 \alpha$ (Figure 2A). More importantly, we validated the existence of EZH2 $\beta$ at the protein level using an affinitypurified antibody specifically generated against this novel isoform (Figure 2B). The electrophoretic mobility of these two isoforms as resolved by SDS-PAGE is in agreement with the molecular weights predicted from the respective amino acid compositions (EZH2 2 : $86.03 \mathrm{kDa}$; EZH2 $8: 81.05 \mathrm{kDa})$. Experiments with these specific antibodies confirmed that the EZH2 $\beta$ transcript is readily translated into a protein that has the potential to function as a novel member of the PRC2 complex (Figure 2C). Furthermore, the pattern of expression of EZH2 $\beta$ as defined by western blot was highly concordant with that predicted by PCR-based transcript analysis with a marked enrichment in testes and ovary and absence from brain. For comparison, we generated an antibody against EZH $2 \alpha$ that, similar to all commercially available tools, primarily detects this protein but has the potential to cross-react with other EZH2 products. Our results demonstrate that our antibodies readily recognize both EZH $2 \alpha$ and $E Z H 2 \beta$ proteins with high specificity (Figure $2 \mathrm{~B}$ ). To our surprise, we did not observe additional bands besides those corresponding to EZH $2 \alpha$ and EZH2 $\beta$, suggesting that, in spite of the large splicing potential of EZH2, these two proteins are the most readily detected products generated from this locus in human cells. Next, given the high degree of expression shown for EZH2 $\beta$ by our transcriptional and proteomic screens, we performed immunohistochemistry against this protein in human testis (Figure 2D), where cell fate determination relies on two pools with different proliferative mechanisms, namely meiosis and mitosis. Interestingly, the EZH2 $\beta$-specific antibody revealed its preferential expression in the primary spermatogonia nuclei (white arrow, Figure 2D, left), a subpopulation of cells that are concentrated along the basal lamina and actively undergoing mitosis. Staining for EZH2 $\beta$ was absent in the nuclei of primary and secondary spermatocytes that are located more centrally in the tubules and divide by meiosis (white circle, Figure 2D, left). This difference in labeling hints at differences in the function of EZH2 $\beta$ versus the total EZH2 pool. Comparatively, the use of a well-characterized EZH $2 \alpha$ antibody revealed immunoreactivity across all cellular types within seminiferous tubules, including in the nuclei of spermatogonia as well as primary and secondary spermatocytes (white circle, Figure 2D, right).

Our combined results demonstrate that the EZH2 locus gives rise to various isoforms, confirms the existence of two major isoforms (EZH2 $\alpha$ and $E Z H 2 \beta)$ at both the mRNA and protein levels, and shows their localization in tissue by immunohistochemistry. These experimental datasets encouraged us to subsequently 


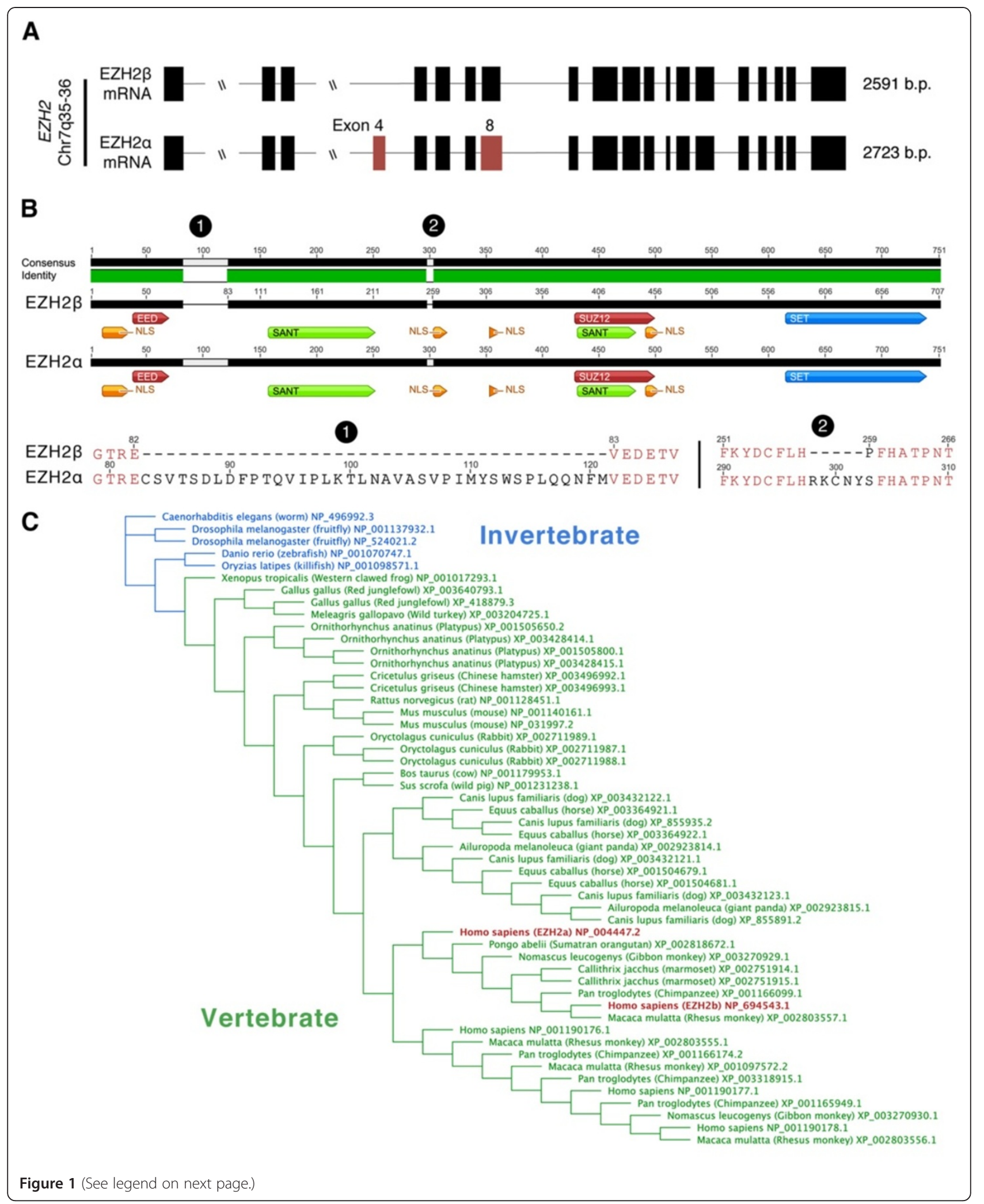


(See figure on previous page.)

Figure 1 The EZH2 locus yields two major transcriptional variants: EZH2a and EZH2 3 . (A) Comparative analysis of the structure of EZH2a and $\mathrm{EZ} 2 \mathrm{H} 2 \beta$ transcript variants where sites of alternative splicing events are highlighted in red on the reference isoform. Details of splicing events are described in Table 1. (B) Protein structure differences between EZH2a and EZH2 $\beta$ proteins shows the conservation of functional domains and binding sites of enzymatic co-factors, represented by different colors and labeled accordingly. Labels 1 and 2 indicate the locations of deletions in $\mathrm{EZH} 2 \beta$ compared with EZH2a. A comparison of the amino acid sequence highlighting the amino acid differences between the two proteins is also presented. (C) Evolutionary dendrogram of invertebrate (blue) and vertebrate (green) EZH2 isoforms. Nodes are spaced according to evolutionary distance. Human $E Z \mathrm{H}_{2} \mathrm{a}$ and $\mathrm{EZ} 2 \mathrm{H} 2 \beta$ are highlighted in red. Combined, these results reveal the potential of EZH2 to generate various isoforms through alternative splicing mechanisms as well as highlight the conservation of EZH2 isoforms throughout evolution. Bp: base pair; EZH2: enhancer of zeste homologue 2.

perform functional studies to test whether these different EZH2 isoforms play redundant or overlapping functions in human cells by analyzing their cellular localization, cofactor binding, and behavior as H3-K27 writers during gene silencing on an isolated gene promoter, as well as their genome-wide effects on gene expression. We also sought to gain insight into their biological function by analyzing their effect on cell proliferation, one of the best-characterized functions attributed to the EZH2 locus in normal morphogenesis and cancer.
EZH2 $\beta$ localizes to the nucleus and interacts with SUZ12 and EED

EZH2 has been historically considered an exclusively nuclear protein, although previous studies have described EZH2 immunoreactivity in the cytoplasm of cancer cells [31]. This knowledge prompted us to better define the cell compartment where the new EZH2 isoform functions using confocal microscopy on isolated epithelial cells. We found that although EZH $2 \beta$ as well as EZH $2 \alpha$ localize to the cell nucleus (Figure $3 \mathrm{~A}$ ), neither of our

Table 1 Comparison of EZH2 $\alpha$ and EZH2 $\beta$ transcripts yielded by alternative splicing of EZH2 locus transcripts

\begin{tabular}{|c|c|c|c|c|c|c|c|c|c|}
\hline \multicolumn{5}{|c|}{ EZH $2 a$} & \multicolumn{5}{|c|}{$E Z H 2 \beta$} \\
\hline Exon & $\begin{array}{c}\text { Exon } \\
\text { size }\end{array}$ & $5^{\prime}$ donor & $3^{\prime}$ acceptor & $\begin{array}{c}\text { Intron } \\
\text { size }\end{array}$ & Exon & $\begin{array}{c}\text { Exon } \\
\text { size }\end{array}$ & $5^{\prime}$ donor & $3^{\prime}$ acceptor & $\begin{array}{c}\text { Intron } \\
\text { size }\end{array}$ \\
\hline 1 & 186 & ACGAAGgtaacgc & cttttagAATAAT & 36,858 & 1 & 186 & ACGAAGgtaacgc & cttttagAATAAT & 36,858 \\
\hline 2 & 124 & GTAAAGgtataat & ttaaagAGTATG & 583 & 2 & 124 & GTAAAGgtataat & ttaaagAGTATG & 583 \\
\hline 3 & 129 & AGGGAGgttggtt & gttttagTGTTCG & 13,719 & 3 & 129 & AGGGAGgttggtt & ttttagGTGGAA & 16,621 \\
\hline 4 & 117 & TTATGgtatgta & ttttagGTGGAA & 2,785 & 4 & 121 & ATAGAGgtgagcc & gtttcagAATGTG & 847 \\
\hline 5 & 121 & ATAGAGgtgagcc & gtttcagAATGTG & 847 & 5 & 141 & GAGATGgtatgcc & tgtttagATAAAG & 1,473 \\
\hline 6 & 141 & GAGATGgtatgcc & tgtttagATAAAG & 1,473 & 6 & 103 & GGAAAAgtaagaa & atgtcagATATAA & 531 \\
\hline 7 & 103 & GGAAAAgtaagaa & atgtcagATATAA & 531 & 7 & 164 & TACATCgtaagt & tttgcagCTाTTC & 6,781 \\
\hline 8 & 179 & ATTATTgtacgtt & tttgcagCTाTTC & 6,766 & 8 & 92 & CATTTGgtaagac & ttcgtagGAGGGA & 1,478 \\
\hline 9 & 92 & CATTTGgtaagac & ttcgtagGAGGGA & 1,478 & 9 & 241 & CCTCTGgtaagac & tttgtagAAGCAA & 485 \\
\hline 10 & 241 & CCTCTGgtaagac & tttgtagAAGCAA & 485 & 10 & 170 & AGACAGgtaaga & ttgtcagGTGTAT & 443 \\
\hline 11 & 170 & AGACAGgtaaga & ttgtcagGTGTAT & 443 & 11 & 95 & ACACCGgtgagtC & tttgcagGTTGTG & 1,137 \\
\hline 12 & 95 & ACACCGgtgagtC & tttgcagGTTGTG & 1,137 & 12 & 41 & AAAAGGgttagca & tactcagACGGCT & 466 \\
\hline 13 & 41 & AAAAGGgttagca & tactcagACGGCT & 466 & 13 & 126 & CAGAGTgtaagta & tctgaagGTCAAA & 776 \\
\hline 14 & 126 & CAGAGTgtaagta & tctgaagGTCAAA & 776 & 14 & 179 & AAAAAGgtgagca & tctctagCATCTA & 2,238 \\
\hline 15 & 179 & AAAAAGgtgagca & tctctagCATCTA & 2,238 & 15 & 96 & GGAGAGgtaagge & tttttagATTATT & 1,210 \\
\hline 16 & 96 & GGAGAGgtaaggc & tttttagATTATT & 1,210 & 16 & 82 & ACAATGgtatgtt & cttttagATाTT & 942 \\
\hline 17 & 82 & ACAATGgtatgtt & cttttagATाTG & 942 & 17 & 81 & CAAAAGgtaggta & tttgcagTTATGA & 154 \\
\hline 18 & 81 & CAAAAGgtaggta & tttgcagTTATGA & 154 & 18 & 85 & TTACAGgttggta & gtttcagATACAG & 1,364 \\
\hline 19 & 85 & TTACAGgttggta & gtttcagATACAG & 1,364 & 19 & 335 & TTGAATCatctctc & ND & \\
\hline 20 & 335 & TTGAATCatctctc & ND & & & & & & \\
\hline
\end{tabular}

Deposited cDNA sequences for EZH2a (NM_004456.4) and EZH2 $\beta$ (NM_152998.2) were aligned against the most recently published human genome sequence (Feb. 2009 GRCh37/hg19) to determine splice donors, splice acceptors, and exon and intron sizes using the University of California, Santa Cruz BLAT tool. ND: not determined. 
Table 2 Conservation of the EZH $2 \alpha$ and EZH2 $\beta$ isoforms across species

\begin{tabular}{|c|c|c|c|c|}
\hline RefSeq & Species & Common name & $\begin{array}{c}\text { EZH2a/NP_004447.2 } \\
\% \text { identity }\end{array}$ & $\begin{array}{c}\text { EZH2 } \beta / N P \_694543.1 \\
\% \text { identity }\end{array}$ \\
\hline NP_004447.2 & Homo sapiens & Human & 100.0 & 94.0 \\
\hline NP_694543.1 & Homo sapiens & Human & 94.0 & 100.0 \\
\hline XP_002923814.1 & Ailuropoda melanoleuca & Giant panda & 98.7 & 92.7 \\
\hline XP_002923815.1 & Ailuropoda melanoleuca & Giant panda & 92.6 & 98.3 \\
\hline NP_001179953.1 & Bos taurus & Cow & 97.6 & 91.8 \\
\hline NP_496992.3 & Caenorhabditis elegans & Worm & 23.8 & 23.4 \\
\hline XP_003496992.1 & Cricetulus griseus & Chinese hamster & 96.6 & 92.3 \\
\hline XP_003496993.1 & Cricetulus griseus & Chinese hamster & 96.2 & 90.3 \\
\hline XP_002751914.1 & Callithrix jacchus & Marmoset & 97.9 & 93.5 \\
\hline XP_002751915.1 & Callithrix jacchus & Marmoset & 92.6 & 98.3 \\
\hline XP_003432121.1 & Canis lupus familiaris & Dog & 97.9 & 93.5 \\
\hline XP_003432122.1 & Canis lupus familiaris & Dog & 96.7 & 92.3 \\
\hline XP_003432123.1 & Canis lupus familiaris & Dog & 92.6 & 98.3 \\
\hline XP_855891.2 & Canis lupus familiaris & Dog & 86.9 & 92.3 \\
\hline XP_855935.2 & Canis lupus familiaris & Dog & 91.0 & 86.5 \\
\hline NP_001137932.1 & Drosophila melanogaster & Fruit fly & 53.6 & 51.9 \\
\hline NP_524021.2 & Drosophila melanogaster & Fruit fly & 53.6 & 51.9 \\
\hline NP_001070747.1 & Danio rerio & Zebrafish & 84.0 & 80.6 \\
\hline XP_001504679.1 & Equus caballus & Horse & 98.7 & 94.2 \\
\hline XP_001504681.1 & Equus caballus & Horse & 93.5 & 99.4 \\
\hline XP_003364921.1 & Equus caballus & Horse & 97.5 & 93.0 \\
\hline XP_003364922.1 & Equus caballus & Horse & 91.7 & 87.3 \\
\hline XP_003640793.1 & Gallus gallus & Red jungle fowl & 91.2 & 97.0 \\
\hline XP_418879.3 & Gallus gallus & Red jungle fowl & 96.1 & 92.0 \\
\hline XP_001097572.2 & Macaca mulatta & Rhesus monkey & 98.0 & 93.6 \\
\hline XP_002803555.1 & Macaca mulatta & Rhesus monkey & 99.2 & 94.8 \\
\hline XP_002803556.1 & Macaca mulatta & Rhesus monkey & 92.3 & 87.8 \\
\hline XP_002803557.1 & Macaca mulatta & Rhesus monkey & 94.0 & 100.0 \\
\hline XP_003204725.1 & Meleagris gallopavo & Wild turkey & 91.0 & 86.8 \\
\hline NP_001140161.1 & Mus musculus & Mouse & 97.1 & 91.3 \\
\hline NP_031997.2 & Mus musculus & Mouse & 97.6 & 93.3 \\
\hline XP_003270929.1 & Nomascus leucogenys & Gibbon monkey & 99.2 & 94.8 \\
\hline XP_003270930.1 & Nomascus leucogenys & Gibbon monkey & 92.3 & 87.8 \\
\hline XP_001505650.2 & Ornithorhynchus anatinus & Platypus & 90.4 & 86.1 \\
\hline XP_001505800.1 & Ornithorhynchus anatinus & Platypus & 92.3 & 98.2 \\
\hline XP_003428414.1 & Ornithorhynchus anatinus & Platypus & 97.3 & 93.0 \\
\hline XP_003428415.1 & Ornithorhynchus anatinus & Platypus & 92.3 & 98.2 \\
\hline XP_002711987.1 & Oryctolagus cuniculus & Rabbit & 98.3 & 93.8 \\
\hline XP_002711988.1 & Oryctolagus cuniculus & Rabbit & 93.1 & 99.0 \\
\hline XP_002711989.1 & Oryctolagus cuniculus & Rabbit & 97.9 & 91.9 \\
\hline NP_001098571.1 & Oryzias latipes & Killifish & 82.0 & 78.6 \\
\hline XP_002818672.1 & Pongo abelii & Sumatran orangutan & 95.5 & 90.7 \\
\hline XP_001165949.1 & Pan troglodytes & Chimpanzee & 92.3 & 87.8 \\
\hline
\end{tabular}


Table 2 Conservation of the EZH2 $\alpha$ and EZH2 $\beta$ isoforms across species (Continued)

\begin{tabular}{lcccc}
\hline XP_001166099.1 & Pan troglodytes & Chimpanzee & 94.0 & 100.0 \\
XP_001166174.2 & Pan troglodytes & Chimpanzee & 99.2 & 94.8 \\
XP_003318915.1 & Pan troglodytes & Chimpanzee & 98.0 & 93.6 \\
NP_001128451.1 & Rattus norvegicus & Rat & 97.5 & 93.2 \\
NP_001231238.1 & Sus scrofa & Wild pig & 96.7 & 92.2 \\
NP_001017293.1 & Xenopus tropicalis & Western clawed frog & 93.2 & 89.0 \\
\hline
\end{tabular}

Human EZH2a and EZH2 $\beta$ were aligned against all predicted EZH2 isoforms across species to determine the degree of conservation. The highest scoring correlations for each species and the two isoforms are indicated. Global alignment with free ends gap was performed using the Geneious alignment tool with BLOSUM62 matrix constrained by an open gap penalty of 12 and gap extension penalty of 3 .

antibodies noticeably labeled the cytoplasm. Thus, although it remains possible that alternative splicing can contribute to the generation of a cytoplasmic form of $\mathrm{EZH} 2$, we found no evidence for this localization with EZH2 $\beta$ under the conditions studied.

We also performed co-immunoprecipitation experiments to define whether EZH2 $\beta$ interacts with other members of the PRC2 complex. We found that this protein is capable of complexing with SUZ12 and EED (Figure 3B), previously described as obligate co-factors of conventional EZH2 methyltransferase function, although the interaction between SUZ12 and EZH2 $\beta$ is seemingly weaker than that between SUZ12 and EZH2 $\alpha$. Thus, the sequences used by various EZH2 proteins for complexing to its enzymatic partners are not only conserved but also functional. Together, the results of these biochemical studies demonstrate that EZH2 $\beta$ shares with EZH2 $\alpha$ the ability to localize to the cell nucleus and complex with SUZ12 and EED, suggesting that both proteins display mechanistic properties that are expected for them to participate in the regulation of gene repression, an idea which was tested at higher stringency in subsequent experiments.

$\mathrm{EZH} 2 \beta$ mediates $\mathrm{H} 3-\mathrm{K} 27$ me3-associated gene silencing on promoters for homeodomain-containing proteins in genomically integrated reporter systems and isolated murine $\mathrm{T}$ cells

The histone code hypothesis represents a useful paradigm for understanding how histone marks deposited by writer proteins (for example, HMTs) recruit mark readers to gene promoters, which triggers the transition between permissive and non-permissive chromatin that ultimately regulates gene transcription. Currently, the conventional $\mathrm{EZH} 2$ protein $(\mathrm{EZH} 2 \alpha)$ is the most characterized writer of the H3-K27me3 mark in organisms ranging from flies to humans. For instance, via this mechanism, EZH2containing PRCs have an evolutionarily conserved role in regulating the expression of entire families of transcriptional regulators, such as those for homeobox, Sry-related high-mobility-group box and forkhead box (FOX) families [33]. The promoter of the human forkhead homologue, FOXP3, has recently been described by our group as a
PRC2-regulated gene containing one of the few identified mammalian Polycomb response elements [34]. Therefore, we used the FOXP3 promoter as a model for assaying the transcriptional regulatory function of EZH2 $\beta$. This system allowed us to test the hypothesis that, similar to EZH $2 \alpha$, $\mathrm{EZH} 2 \beta$ fulfills the criteria as a writer of the H3-K27me3 known to precipitate gene silencing. Because EZH2 function associates with long-term silencing, instead of using the typical episomally based reporter systems, we used an integrated luciferase gene system in which the FOXP3 promoter was cloned after the cytomegalovirus (CMV) promoter (JFOXP3-FLP). This design allowed us to measure the dominant effects of EZH2-mediated silencing effects over the robust activation provided CMV promoter in a highly sensitive integrated system.

Our experiments demonstrated that EZH2 $\beta$ alone or when combined with obligate PRC2 complex partners (SUZ12 and EED) significantly represses luciferase activity in JFOXP3-E1 FLP compared to transfection with empty vector alone. Compared with empty vector, EZH2 $\beta$ had luciferase expression of $26.53 \pm 8.53 \%$ when alone and $28.60 \pm 17.23 \%$ when in complex, which is equivalent to a $73.7 \%$ reduction in promoter activity when alone. $\mathrm{EZH} 2 \alpha$ was included in these experiments as a comparison and also repressed luciferase expression. EZH $2 \alpha$ had luciferase expression of $45.97 \pm 25.45 \%$ and $24.46 \pm 2.36 \%$ compared with empty vector, alone and in complex, respectively (Figure 4A), corresponding to a 54.03\% reduction in promoter activity when alone. Thus, using this engineered cell reporter system, we conclude that, in vitro, EZH2 $\beta$ displays key functional properties that are expected from a histone code writer. Equally important, these results demonstrate that various EZH2 proteins can achieve the gene silencing function previously attributed to a single HMT protein.

In light of these results, we subsequently sought to gain insight as to whether this process is also operational in vivo in primary cells by evaluating the role of EZH2 $\beta$ in the regulation of FOXP3 expression in isolated murine $\mathrm{T}$ lymphocytes. As these primary cells are notoriously difficult to transfect or infect with most of the viruses used for ex vivo gene transfer, we isolated 


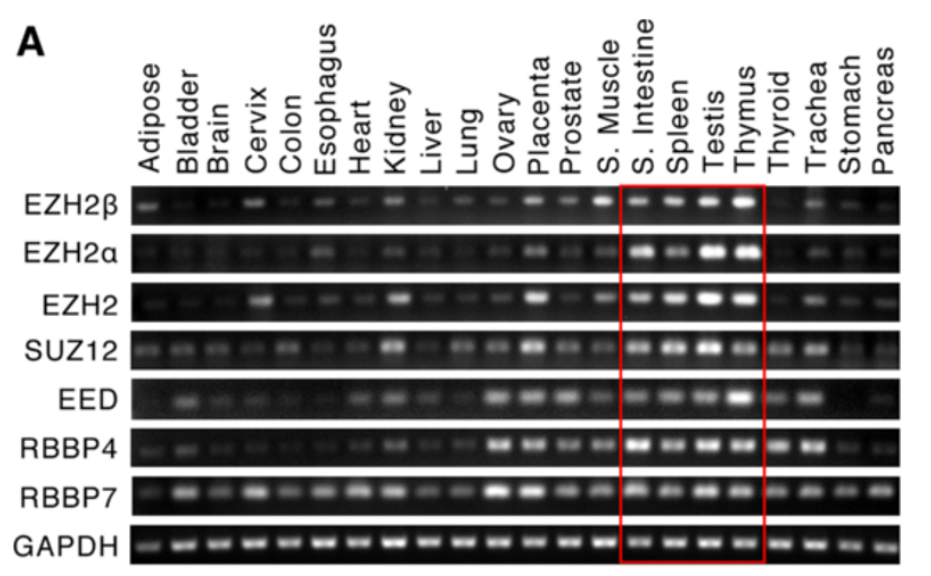

B

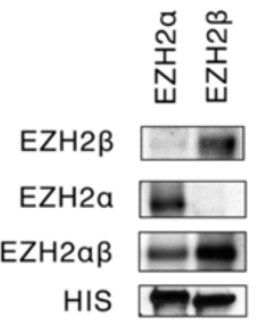

C

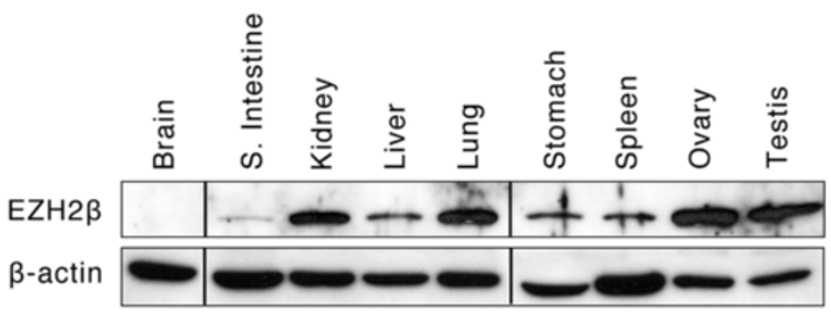

D

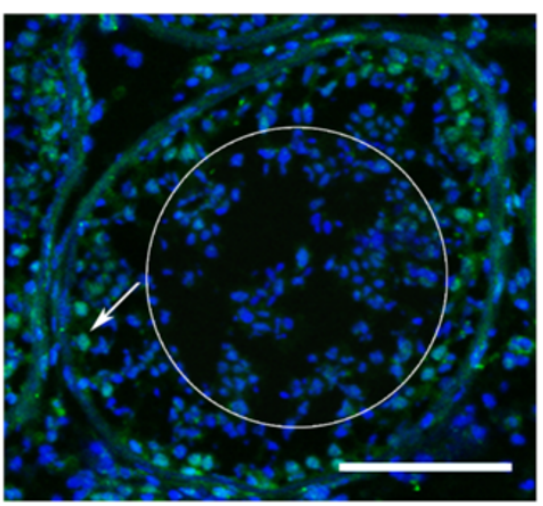

$\mathrm{EZH} 2 \beta$

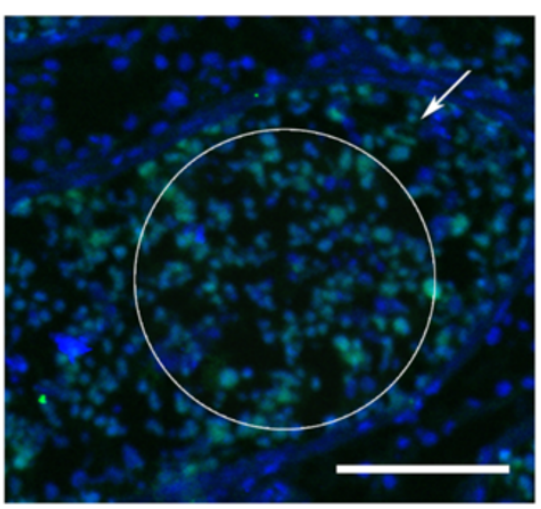

$\mathrm{EZH} 2$

Figure 2 EZH2 $\beta$ is expressed in a variety of adult human tissues. (A) Analysis of EZH2 $\beta, E Z H 2 a$, as well as associated complex co-factors SUZ12, EED, RBBP4 and RBBP7 transcripts in 22 human tissues. RT-PCR was performed with primers designed to differentiate the two EZH2 isoforms. Analysis of glyceraldehyde-3-phosphate dehydrogenase was used as amplification control. Red box indicates tissues that possess highly proliferative capacity and preferential co-expression of all PRC2 complex transcripts, including EZH2 $\beta$. (B) Specificity of EZH2a and EZH2 $\beta$ antibodies. Isoform-specific antibodies were tested using western blot of untransfected pancreatic cancer cell lines (Additional file 1: Figure S1) and also shown here in cells transfected with histidine (HIS) epitope-tagged EZH2a, EZH2 3 or both isoforms (EZH2aß). Labeling of the HIS-tag was used as loading control. (C) Tissue distribution of EZH2 $\beta$ at the protein level where the presence of $\mathrm{EZH} 2 \beta$ was determined by western blot analysis of human tissues with the EZH2 $\beta$-specific antibody. $\beta$-actin was used as loading control. (D) Fluorescent immunohistochemistry of samples of frozen human testis was performed by laser confocal microscopy, in sections labeled for EZH2 (left) and total EZH2 (right). The white circle encompasses primary and secondary spermatocytes, whereas primary spermatogonia are immediately adjacent to the basal lamina (white arrow). Nuclei are counterstained with Hoechst. Images were obtained at 10x magnification. White scale bar represents $100 \mu \mathrm{m}$. Together, these results validate that the two major isoforms generated by the human EZH2 locus, namely EZH2a and EZH2 $\beta$, are translated into proteins that can be detected not only in cell lysates but also in whole tissues. EED: embryonic ectoderm development; EZH2: enhancer of zeste homologue 2; GADPH: glyceraldehyde-3-phosphate dehydrogenase; HIS: histidine; RBBP: retinoblastoma binding protein; SUZ12: suppressor of zeste 12. 
lymphocytes from a mouse line transgenically expressing the adenoviral receptor (CAR transgenic mouse, Taconic, model 4285) that are amenable to adenoviral-mediated transduction. Thus, naïve CD4+ splenocytes were isolated from the CAR transgenic mouse and infected with EZH2 $\beta$, EZH $2 \alpha$ or control empty adenoviruses. Primary naïve murine CD4+ lymphocytes transduced with EZH2 $\beta$ did not express FOXP3 upon stimulation when compared with cells transduced with empty vector (Figure $4 \mathrm{~B}$ and Additional file 2: Figure S2), indicating that recruitment of EZH2 to the FOXP3 core promoter results in specific and persistent silencing of FOXP3 expression. This result was also observed for EZH $2 \alpha$. Compared with $17.6 \pm 3.12 \%$ of FOXP3-expressing cells under control conditions, EZH $2 \beta$ overexpression reduced the number of FOXP3expressing cells to $3.26 \pm 0.94 \%$, and EZH $2 \alpha$ reduced this population to $4.28 \pm 0.58 \%$. Complementary qPCR assay detected a reduction of FOXP3 transcription of $45.1 \pm 16.7 \%$ by EZH $2 \beta$ and $26.9 \pm 6.9 \%$ by EZH $2 \alpha$ compared with empty vector (Figure $4 \mathrm{C}$ ). Furthermore, in these experiments, EZH2 $\beta$ overexpression led to increased levels of EZH2 $\beta$ and $\mathrm{H} 3-\mathrm{K} 27 \mathrm{me} 3$ bound to the FOXP3 core promoter, which was also found with EZH $2 \alpha$ overexpression (Figure 4D).

Through the use of two well-defined systems specially engineered to analyze EZH2-mediated gene silencing in lymphocytes (Jurkat-FLP and primary CD4+ splenocytes), we demonstrate that $\mathrm{EZH} 2 \beta$ is capable of gene repression that is mediated by trimethylation of $\mathrm{H} 3-\mathrm{K} 27$, indicating that EZH2 $\beta$ behaves as a histone code writer in a manner which is highly similar to the conventional EZH $2 \alpha$ protein. These results suggest that both EZH2 proteins share mechanisms and potentially regulate similar cellular processes and gene targets. Thus, we tested these ideas by first performing functional cell assays, and subsequently, through the generation of genome-wide expression profiles for these EZH2 proteins.

\section{Expression of EZH2 $\beta$ stimulates cellular proliferation}

$\mathrm{EZH} 2$ is among the best-characterized epigenetic regulators which, when overexpressed, increases proliferation and functions as an oncogene. Consequently, we investigated whether the new EZH2 $\beta$ isoform is functional in cell biological assays using cell proliferation as a model. We performed these experiments in naive primary lymphocytes transduced with empty vector, EZH2 $\beta$, or EZH2 $\alpha$. Figure 4e shows that overexpression of EZH $2 \beta$ and EZH $2 \alpha$ results in an increase in cellular proliferation compared with the control empty vector. This functional analysis is congruent with the localization of these proteins to actively proliferating cell populations (Figure 2D) and with our data from genome-wide expression analyses, shown below, which support that both EZH2 isoforms regulate pro-proliferative gene targets. Taken together, these data indicate that EZH2 $\beta$ is functional in well-established cell biological assays.

\section{Expression of $\mathrm{EZH} 2 \beta$ gives rise to a unique genome-wide transcriptional profile}

Expression profile experiments offer a genome-wide level reporter assay to investigate whether EZH2 $\alpha$ and EZH2 $\beta$ possess common or divergent functions. This experiment was chosen because EZH $2 \beta$ expression follows, in most cases, the expression pattern of EZH $2 \alpha$ in the majority of tissue types studied. EZH2 is a known oncogene for a large number of tissues, including pancreatic cancer. Thus, we used a pancreatic epithelial cell system combined with adenoviral-mediated introduction to overexpress each isoform in an attempt to model the effects of pathological overexpression of each EZH2 isoform on gene repression. For this purpose, we performed Affymetrix GeneChip Human Gene 1.0 ST arrays, which showed that of the 28,869 well-annotated genes assessed, 366 unique targets (36.3\% of total repressed) were uniquely repressed by EZH2 $\beta\left(P<0.05\right.$ and $\log _{2}$ fold change $>-2$ for EZH2 $\beta$, $P>0.05$ for $\mathrm{EZH} 2 \alpha$, Figure 5). EZH $2 \alpha$-generated expression profiles displayed a downregulation of 480 targets $\left(47.6 \%\right.$ of total repressed, $P<0.05$ and $\log _{2}$ fold change $>-2$ for EZH2 $\alpha, P>0.05$ for EZH2 $\beta$, Figure 5$)$. Notably, 162 targets (16.1\% of total repressed) were repressed by both EZH $2 \alpha$ and EZH2 $\beta$ (Figure $5, P<0.05, \log _{2}$ fold change $>-2$ for both). Both isoforms also induced upregulation in the expression of a significant number of targets, which may reflect indirect effects mediated by the repression of upstream regulators. Of the genes assayed, 444 (39.6\% of total activated) were activated by EZH2 $\beta$, $382(34 \%)$ by EZH2 $\alpha$ and $296(26.4 \%)$ by both isoforms. Therefore, as demonstrated by the overall array data, the novel EZH2 $\beta$ isoform described here is responsible for the expression pattern of its own unique set of genes, in addition to a group of common targets with EZH $2 \alpha$.

Repressed genes were organized into ontological categories using Ingenuity Pathways Analysis (IPA)-based classifications (Figure 5B). Notably, EZH2 $\beta$ was found to regulate genes involved in key cellular functions including proliferation, differentiation and angiogenesis, which were previously attributed only to EZH2 isoform. This concept was better visualized by cross-validating our expression data with a subset of canonical EZH2-regulated targets as identified by a previously reported EZH2 chromatin immunoprecipitation-sequencing (ChIP-seq) dataset in a different cell line [35], generated using an antibody that, according to our data, recognizes both the EZH $2 \alpha$ and $\mathrm{EZH} 2 \beta$ isoforms. Cross-reference of significantly repressed and activated genes parsed from our genome-wide expression data (Figure 5C) with this independent subset of targets demonstrates a division pattern similar to that observed in the transcriptional profiles. As such, from a large 


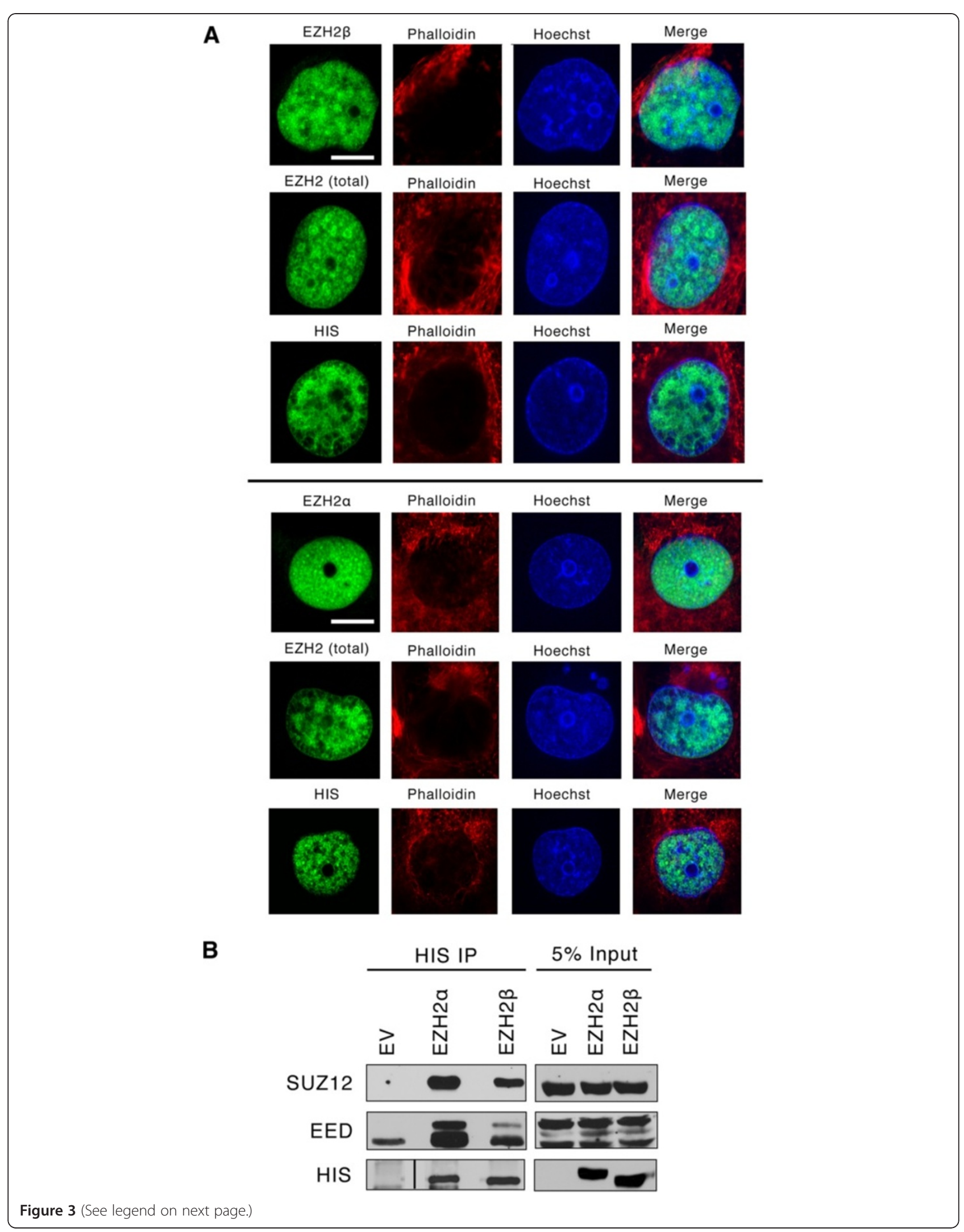


(See figure on previous page.)

Figure $3 \mathrm{EZH} 2 \beta$ is localized to the nucleus and partners with SUZ12 and EED. (A) Subcellular localization of EZH2 $\beta$. Antibodies against total $E Z H 2, E Z H 2 a$ or $E Z H 2 \beta$ were used to determine $E Z H 2 a$ or $E Z H 2 \beta$ localization by immunofluorescence of epithelial cells transduced with HIS/EZH2 $\beta$ or HIS/EZH2a. Labeling of the HIS-tag was performed to both confirm localization and control for expression. Nuclei are counterstained with Hoechst and cytoskeletal components labeled with phalloidin red. Images were taken at 100x magnification. White scale bar represents $10 \mu \mathrm{m}$. (B) EZH2 $\beta$ interaction with SUZ12 and EED. Immunoprecipitation from whole cell extracts harvested from epithelial cells transduced with HIS/EZH2a or HIS/EZH2 $\beta$ using an antibody against the HIS-tag were probed with antibodies against SUZ12 and EED. Five percent inputs of whole cell lysates were included as control of transduction and expression. These results demonstrate that EZH2 $\beta$ localizes to the nucleus and interact with PRC2 targets, which are two key features expected of a functional EZH2 isoform. EED: embryonic ectoderm development; HIS: histidine; EZH2: enhancer of zeste homologue 2; EV: empty vector; immunoprecipitation; SUZ12: suppressor of zeste 12.

subset of genes previously thought to be regulated by a single EZH2 HMT, we determined the overlap between the isoform-specific targets we identified and this subset of canonical EZH2 targets. Figure 5A-right depicts both occupancy and expression measured on a subset of wellvalidated canonical Polycomb targets that have previously been shown to be regulated by EZH $2 \alpha$. The box highlights a subset of targets that were identified as EZH2 $\beta$ or EZH2 $\alpha$ specific from our whole genome assay (Figure 5A-left). Thus, although each isoform possesses similarity in terms of ontological functions, mediation of these functions appears to be executed through the repression of primarily unique, although sometimes common, targets.

IPA-based network analysis identified a number of subnetworks of interdependent genes enriched for particular functions and/or participation in disease processes. EZH2 $\beta$, for instance, was able to uniquely repress a subnetwork enriched for functions in cellular maintenance and function as well as hematological system development and function (Figure 6A). Overexpression of EZH $2 \alpha$, however, led to no significant alteration of these targets. EZH $2 \alpha$ overexpression resulted in the significant repression of a subnetwork of targets that associates to the regulation of cellular growth, cell cycle and proliferation (Figure 6B). Again, EZH2 $\alpha$ repressed many of these targets uniquely without apparent contribution from EZH2 $\beta$. However, subnetwork enrichment for function in cell death survival displayed equal repression by either isoform (Figure 6C). Thus, these data indicate that although biochemically quite similar at the level of nuclear localization, transcription and interaction with critical cofactors, each isoform displays a preferential gene expression pattern, which, according to our ontological analyses, supports their participation in a large number of shared biological functions.

\section{Discussion}

The human $E Z H 2$ gene was originally isolated in a screen for proteins which interact with Vav1, a human protooncogene [36,37]. Notably, although most Polycomb functions have been attributed to require the enzymatic activity of PRC2, recent data indicate that other related enzymes may possess redundant or overlapping functions with EZH2, such as EZH1 [10,38]. Despite these advances, most
Polycomb experiments are designed with the paradigm that EZH $2 \alpha$ is the sole H3-K27me3 methyltransferase. Thus, it has become essential to explore the isoform complexity of the EZH2 family of proteins. Consequently, the goal of the current study has been to address this important gap in the existing knowledge.

Drosophila possess only one $\mathrm{E}(\mathrm{z})$ gene, whereas vertebrates possess two paralogs: EZH1 and EZH2, with gene duplication occurring early in evolution, as two paralogs have been identified in zebrafish [10]. Evidence for alternative splicing is evident even in the ancestral $\mathrm{E}(\mathrm{z})$ gene with observed expansions and reductions in the progression from invertebrate to vertebrate. Although gene duplication of HMT genes, as observed with Ez proteins and other HMTs, such as Suv4-20 h1/h2 and Suv39h1/h2 [39], appear to serve redundant functions, the early expansion of EZH2 through alternative splicing hints at a neofunctionalization phenomenon. The preservation of alternative splicing events from invertebrates to vertebrates supports an evolutionary model in which pressures were high to maintain a diverse pool of EZH2 proteins to facilitate precise regulation of repressive programs.

We have characterized the alternative splicing and translation of the $E Z H 2$ locus to yield a minimum of two distinct functional HMTs: EZH2 $\alpha$, a known enzyme, and EZH2 $\beta$, a new enzyme. Biochemical characterization of EZH2 $\beta$ indicates that it exhibits a similar tissue expression pattern as EZH $2 \alpha$ and that this isoform is widely expressed in human tissues with particularly high levels of expression in tissues dependent on replenishment from a progenitor pool of multipotent cells, such as the thymus and testes. Multiple EZH2-positive bands have been observed by Southern and western blot in previous studies $[25,40,41]$, but were often labeled as artifact. However, our investigation is the first to positively confirm and characterize two distinct isoforms using antibodies designed to distinguish between each protein. Furthermore, we demonstrate that EZH $2 \beta$ is localized exclusively to the nucleus and capable of partnering with obligate Polycomb co-factors SUZ12 and EED to form the minimal PRC2 complex necessary to permit enzymatic activity of the protein. Since the identification of its mammalian homologue, a number of EZH2 transcripts have been identified by genomic 
A

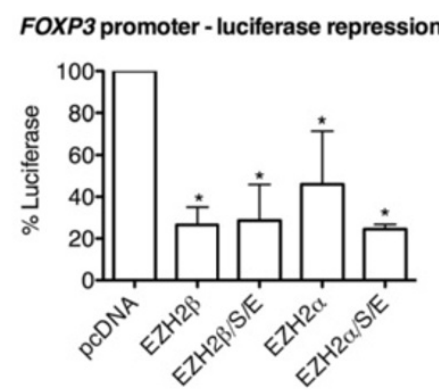

B

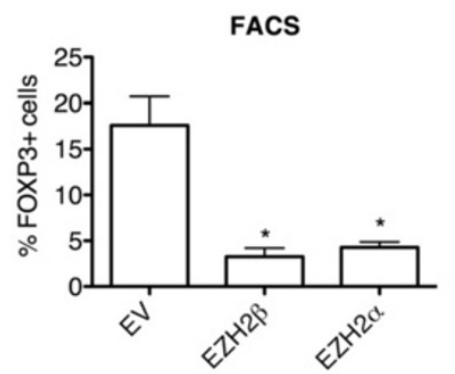

D
C

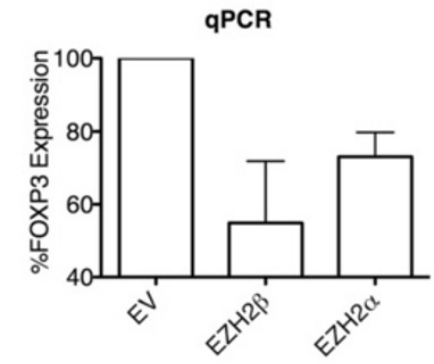

FOXP3 promoter - ChIP
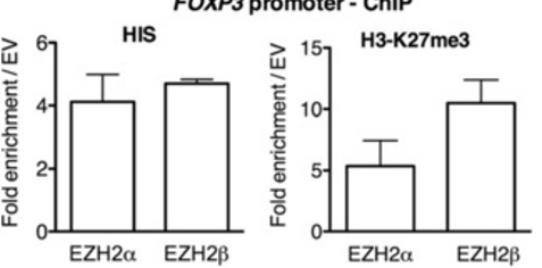

E

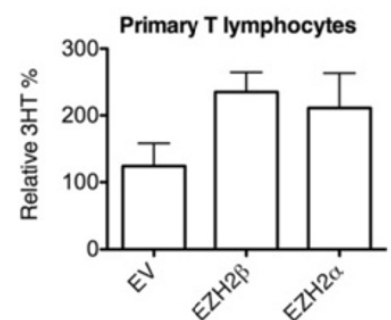

Figure $4 \mathrm{EZH} 2 \beta$ represses transcription through $\mathrm{H} 3-\mathrm{K} 27$ trimethylation of gene promoters and increases cellular proliferation.

(A) Luciferase values are shown relative to control (pcDNA) upon nucleofection of EZH2 $\beta$ or with co-factors SUZ12 and EED (EZH2 $\beta / S / E)$ in cells with an integrated FOXP3 luciferase reporter. EZH2a alone or with co-factors SUZ12 and EED (EZH2a/S/E) is included. ${ }^{*} P<0.05$. (B) Quantification of flow cytometry analysis of primary mouse naïv $T$ cells transduced with empty vector, EZH2 $\beta$ or EZH2a for FOXP3 expression. ${ }^{*} P<0.05$. (C) qPCR of FOXP3 expression in primary mouse naïve T cells indicates that transcription is reduced by transduction of EZH2 $\beta$ and EZH2a. Glyceraldehyde-3-phosphate dehydrogenase and hypoxanthine phosphoribosyltransferase were used as expression controls. (D) ChIP assay of H3-K27me3 on the FOXP3 promoter in primary mouse naïve T cells. Transduction with EZH2a or EZH2 $\beta$ increases H3-K27me3 on the FOXP3 promoter relative to empty vector control. ChIP performed using an antibody against the HIS-tag demonstrates that only the EZH2a- and EZH2 3 -infected cells amplify a band to indicate their presence on the FOXP3 promoter, whereas empty vector-infected cells serve as a negative control. A representative gel is shown from triplicate experiments with associated qPCR quantification. These results reveal that novel EZH2 isoforms can regulate gene expression through $\mathrm{H} 3-\mathrm{K} 27$ trimethylation of gene promoters. (E) Incorporation of ${ }^{3} \mathrm{H}$-thymidine in primary mouse naïve T cells transduced with empty vector, EZH2a or EZH2 $\beta$ after 5 days of stimulation. Representative data shown from experiments performed in duplicate, representing the mean and SD of technical triplicates. These results indicate that EZH2 $\beta$ increases cellular proliferation in a similar fashion as EZH2a. 3HT: ${ }^{3} \mathrm{H}$-thymidine; ChIP: chromatin immunoprecipitation; EV: empty vector; EZH2: enhancer of zeste homologue 2; FACS: fluorescence-activated cell sorting; H3-K27me3: trimethylation of histone 3 at lysine 27; HIS: histidine; qPCR: quantitative polymerase chain reaction. 


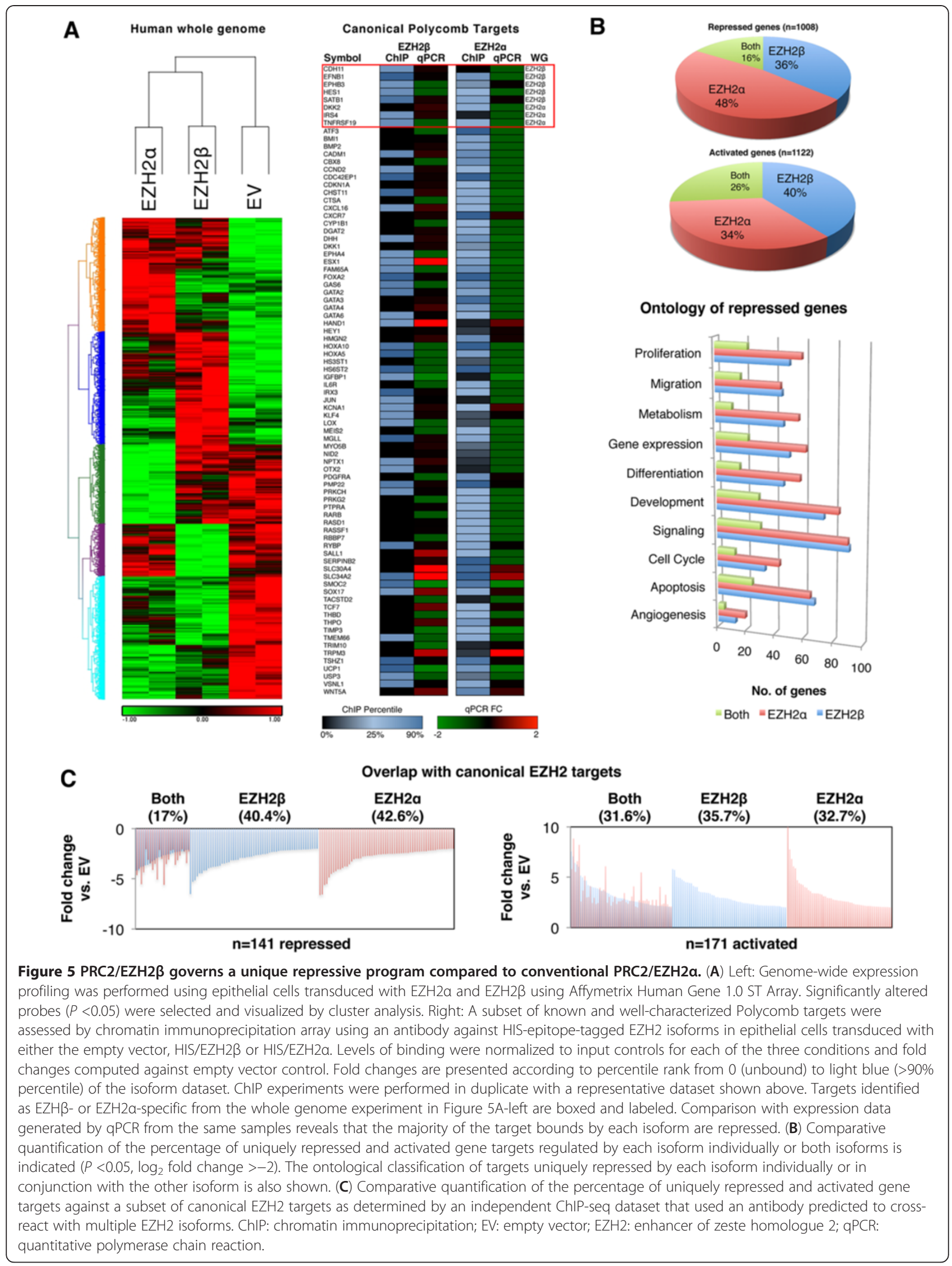




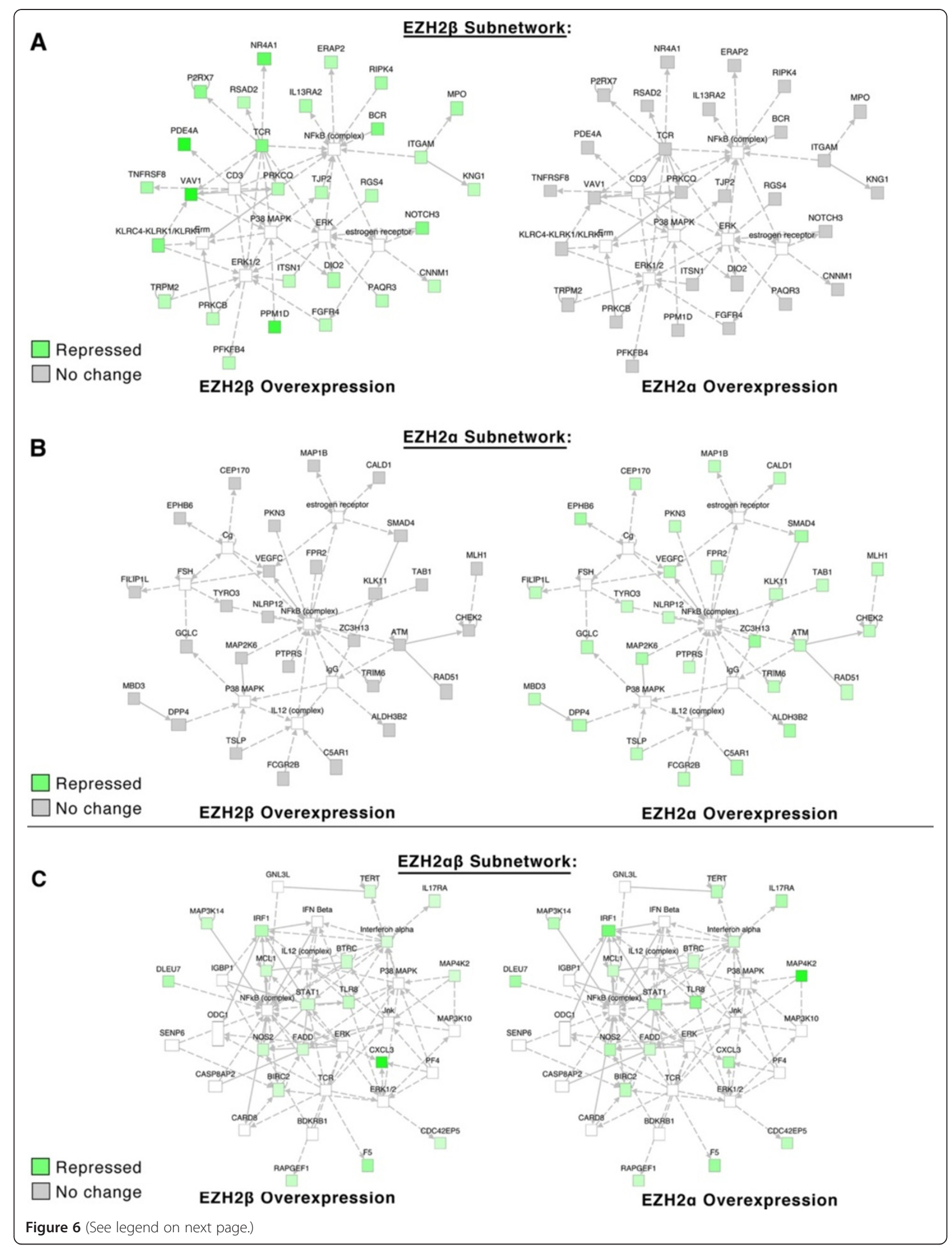


(See figure on previous page.)

Figure $6 \mathrm{EZH} 2$ isoforms can regulate gene expression genome-wide through defined subnetworks. To test if EZH2 $\beta$ - or EZH $2 a-s p e c i f i c$ genes form interdependent, unique subnetworks, Affymetrix data generated in A was parsed for targets uniquely and significantly repressed ( $P<0.05, \log 2$ fold change $>-2$ ) by EZH2 $\beta, E Z H 2 a$ or both compared with empty vector. No change is defined as $P>0.05$ and log 2 fold change between 1.5 and -1.5 . Subnetworks were reconstructed using an IPA-propriety algorithm. While a multitude of subnetworks were generated, a high scoring representative example network for each condition is shown. (A) IPA-assisted subnetwork analysis indicates that EZH2 $\beta$ participates in the regulation of genes involved in several ontological categories, including cellular function and maintenance as well as hematological system function. Overexpression of EZH2 $\beta$ results in significant repression whereas EZH2a overexpression fails to produce the same repressive effects in this particular subnetwork. (B) Similar subnetwork analysis of expression data for enrichments of biological function mediated by EZH2a indicates that this protein regulates targets associated with proliferative responses and cell cycle regulation. (C) Subnetwork analysis of expression data for enrichments of biological function mediated by both $\mathrm{EZH} 2 \beta$ and $\mathrm{EZH} 2 \mathrm{a}$ indicates enrichment of targets involved in cell death and survival and cell signaling. Combined, these data demonstrate that that novel EZH2 isoforms can regulate gene expression genome-wide through unique and shared targets that are interconnected to form defined subnetworks. Note that, although both EZH2 isoforms can often regulate different genes represented by the examples (A, B and C), the subnetworks formed by these genes are ontologically known to participate in similar processes (B). This knowledge not only is congruent with the ability of both $\mathrm{EZH} 2$ proteins to regulate cell growth as revealed by our cell biology assays (Figure $4 \mathrm{E})$, but also expands the potential functional association of these isoforms. EZH2: enhancer of zeste homologue 2.

sequencing efforts, supporting the existence of a family of EZH2 proteins that mediate mammalian gene repression. Extensive future characterization will be required to determine the precise role of each protein variant in gene repression.

PRC2-EZH1 and PRC2-EZH2 $\alpha$ regulate a largely overlapping set of genes, albeit through different mechanisms; PRC2-EZH1 possesses greatly reduced HMT activity compared with PRC2-EZH2 $\alpha$ [10]. Both EZH $2 \alpha$ and EZH2 $\beta$ are capable of repressing FOXP3 expression in vitro, in a manner that is increased by transfection with obligate cofactors SUZ12 and EED. More importantly, using primary mouse naïve $\mathrm{T}$ cells, we demonstrate that both isoforms are able to occupy the FOXP3 promoter with resultant increases in H3-K27me3 and repression of FOXP3 expression, suggesting identical mechanisms of repression. These results highlight the importance for future studies to consider the relative contributions of both isoforms in the regulation of gene repression.

We demonstrate that EZH2 $\beta$ represses a predominantly unique subset of gene targets from EZH2 $\alpha$ with a much smaller percentage of redundant targets than observed between EZH paralogs EZH1 and EZH2 [10]. Although ontology reveals that both isoforms participate in a similar repertoire of biological processes, subnetwork analysis of significantly repressed genes indicates that each isoform regulates distinctive gene networks within process categories. Furthermore, comparison of EZH $2 \alpha$ and EZH $2 \beta$ targets with published ChIP-seq data performed with an antibody that fails to discriminate between isoforms reveals a similar pattern as gene expression data, with each isoform possessing a larger subset of unique rather than redundant targets [35]. The primary difference between the two isoforms is the 39 amino acid insert absent in EZH2 $\beta$ compared with EZH2 $\alpha$. Examination of this insert reveals the presence of potential sites of post-translational modification, including an (ST)-Q motif, which have the potential to be targeted by kinases that participate in a variety of cellular processes including DNA replication and repair. Thus, these data serve as the foundation for future studies aimed at investigating how post-translational modifications can contribute to impart functional specificity of each isoform. Coupled with biochemical data, these studies indicate that EZH $2 \alpha$ and EZH2 $\beta$ are capable of forming distinct repressive complexes that mediate the repression of unique gene networks within a wide variety of biological processes already characterized for PRC2, including proliferation, migration and differentiation, among others [42].

Whole genome gene expression data reveals enrichment for cell cycle and proliferation targets. Overexpression of either isoform in naïve $\mathrm{T}$ cells results in increased cellular proliferation. Additionally, immunohistochemistry of total EZH2 versus EZH2 $\beta$ reveals that EZH2 $\beta$ is localized primarily to developing spermatogonia whereas total EZH2 expression is localized throughout the spermatogonia and spermatocytes. As spermatogonia undergo mitosis, compared to the meiosis occurring in spermatocytes, a potential role for EZH $2 \beta$ in the regulation of cell cycle transitions is likely [43]. Thus, our studies offer a solid rational and build the trajectory for future careful studies aimed at deciphering the role of EZH2 isoforms at the G1/S and G2/M transition points, as well as the type of post-translational modifications, that can regulate these processes.

\section{Conclusions}

Thus far, the functions of EZH2 have been ascribed entirely to isoform EZH2 $\alpha$. The current body of literature will require revision to address the relative contribution of EZH2 isoforms to the biochemical, cellular and pathobiological functions under study. Furthermore, the contribution of alternative splicing to the regulation of HTMs and their function furthers our understanding of the complexity of regulatory mechanisms underlying the 
operation of the histone code. As a result of these findings, a new paradigm of Polycomb-mediated repression must be considered in which cells are armed with a multitude of repressive complexes to regulate distinct gene networks, exponentially increasing the plasticity of the system to meet the broad spectrum of functions required in development, growth and maintenance of biological systems.

\section{Methods}

\section{Plasmids and recombinant adenovirus}

The search for EZH2-related proteins was performed by comparing the human EZH2 SET domain protein sequence (GenBank: BC010858) against the Expressed Sequence Tag database using the BLAST and the UniGene programs from the National Center for Biotechnology Information (National Institutes of Health, Bethesda, MD, USA). This comparison indicated the presence of the EZH2 $\beta$-encoding sequence (NCBI: NM_152998.2). The exact sequences matching this entry as well as other PRC2 proteins, such as SUZ12 (GenBank: BC015704) and EED (GenBank: BC068995), were verified by sequencing and analysis of publically deposited cDNAs. Standard molecular biology techniques were used to clone full-length EZH2 $\alpha, \mathrm{EZH} 2 \beta$, SUZ12 and EED into pcDNA3.1/HIS (Invitrogen, Carlsbad, CA, USA). All constructs were verified by sequencing at the Mayo Clinic Molecular Biology Core Facility. QuickChange Site-Directed Mutagenesis was performed as suggested by the manufacturer (Agilent Technologies, Santa Clara, CA, USA). Silent mutations were made to delete endogenous HindIII and XbaI restriction enzyme sites to permit passage of EZH $2 \alpha$ and EZH2 $\beta$ cDNAs into pacAd5 CMV $\mathrm{K}-\mathrm{N}$ pa shuttle vector. Epitope-tagged (6XHis-Xpress) $\mathrm{EZH} 2 \alpha$ and EZH2 $\beta$ were generated as recombinant adenoviruses by the Gene Transfer Vector Core at the University of Iowa. Empty vector (pacAD5 CMV) was used as the experimental control.

\section{Human tissue RNA panel}

Human total RNA for 22 major organs and tissues was commercially obtained from Ambion (Austin, TX, USA) and Stratagene (Agilent). cDNA was generated from $1 \mu \mathrm{g}$ RNA using SuperScript ${ }^{\mathrm{T}}$ III enzyme (Invitrogen) according to manufacturer's instructions. cDNA concentrations were assessed via internal housekeeping gene glyceraldehyde-3phosphate dehydrogenase or hypoxanthine phosphoribosyltransferase. PCRs were performed with the following cycle conditions: 30 to 35 cycles of $94^{\circ} \mathrm{C}$ for $15 \mathrm{~s}, 50^{\circ} \mathrm{C}$ for $30 \mathrm{~s}$, and $72^{\circ} \mathrm{C}$ for 2 min using 1 to $2 \mu \mathrm{l}$ of cDNA product. Amplified products were electrophoresed on 1.5\% agarose gels, digitally imaged, and quantified with ImageJ (National Institutes of Health, Bethesda, MD, USA). Primers were synthesized by Integrated DNA Technologies (Coraville,
IA, USA). PCR primers may be found in Additional file 3: Table S1.

\section{Western blot analysis}

Samples were run on $4 \%$ to $20 \%$ (Lonza, Walkersville, MD, USA), $6 \%$ or $10 \%$ SDS-PAGE gels and electroblotted onto polyvinylidene difluoride membranes (Millipore, Billerica, MA, USA). The membranes were blocked in 5\% bovine serum albumin or milk in Tris buffered saline with Tween (TBST) for $1 \mathrm{~h}$ at room temperature. The blots were incubated overnight at $4^{\circ} \mathrm{C}$ with primary antibody. After repeated washes in TBST, horse radish peroxidase conjugated anti-rabbit or mouse IgG secondary antibody $(1: 2,000$ to 5,000$)$ was added for $1 \mathrm{~h}$ at room temperature. Blots were developed by Pierce ECL Chemiluminescent Substrate (Thermo Scientific, Rockford, IL, USA). Human tissue lysates were procured from Calbiochem (Millipore) as a ready-to-probe INSTA-blot. Approximately $20 \mu \mathrm{g}$ of lysate was loaded per tissue with loading controlled via amido black straining by the manufacturer. The blot was incubated overnight with EZH2 $\beta$ (purified, 1:2,000) and subsequently stripped and re-incubated with $\beta$-actin (1:1,000; Sigma, St. Louis, MO, USA).

\section{Synthesis, purification and validation of $\mathrm{EZH} 2 \mathrm{a}$ and $\mathrm{EZH} 2 \beta$ antibodies}

A 21-mer peptide bridging across the large insert region missing from EZH $2 \beta$ compared to EZH $2 \alpha$ was synthesized, high performance liquid chromatography-purified and conjugated to keyhole limpet hemocyanin by the Mayo Clinic Protein Core. For the EZH $2 \alpha$ antibody, a 21-mer peptide was synthesized that localized to the insert region. Subsequently, a rabbit was immunized with the peptide, and test and final bleeds were performed by Cocalico Biologicals (Reamstown, PA, USA). For the antibody that recognizes both EZH $2 \alpha$ and EZH $2 \beta$, a 21-met peptide in a region conserved between the two proteins was synthesized. The anti-serum was affinity purified using the Protein A IgG Purification Kit according to the manufacturer's protocol (Pierce Biotechnology, Rockford, IL, USA). To test the specificity of the antibodies, Chinese hamster ovary epithelial cells were transfected with a histidine-tagged (HIS)/EZH $2 \alpha$ and HIS/EZH2 $\beta$. Whole cell lysates $(30 \mu \mathrm{l})$ and pancreatic cell lines $(30 \mu \mathrm{g})$ were resolved on $4 \%$ to $20 \%$ SDSPAGE gels, and probed with whole sera of EZH $2 \alpha$ (1:200), EZH2 $\beta$ (1:200) and EZH $2 \alpha \beta$ (1:200). Blots were stripped and re-probed with Omni-probe (D-8) (1:1,000; Santa Cruz Biotechnology, Santa Cruz, CA, USA) to ensure equal loading.

\section{Immunoprecipitation}

Panc1 epithelial cells were plated at a cell density of $1 \times 10^{6}$ cells/100 mm dish and transduced with epitope-tagged 
(6XHis-Xpress) EZH2 $\alpha, \mathrm{EZH} 2 \beta$ or empty vector at multiplicity of infection (MOI) 150. Subconfluent cells were lysed in a buffer containing $20 \mathrm{mM}$ Tris- $\mathrm{Cl}$ at $\mathrm{pH} 8.0,100$ $\mathrm{mM} \mathrm{NaCl}, 1 \mathrm{mM}$ EDTA, $0.5 \%$ Nonidet P-40 and a protease inhibitor tablet (Roche, San Francisco, CA, USA). Proteins were immunoprecipitated as previously described using $10 \mu \mathrm{g}$ of Omni-probe (D-8) (Santa Cruz Biotechnology) [44]. Resulting complexes were resolved on a $6 \%$ or $10 \%$ SDS-PAGE gels, using antibodies against SUZ12 (1:1,000; Cell Signaling, Beverly, MA, USA) and EED (1:1,000; Cell Signaling). Membranes were stripped and incubated with Omni-probe (D-8) (1:1,000; Santa Cruz), to ensure equal loading of precipitated EZH2 proteins. A 5\% input control of whole cell lysates under all conditions was included to ensure the presence of uniform levels of the proteins of interest.

\section{Cell culture, immunofluorescence and confocal microscopy}

Cell lines were obtained from the American Type Culture Collection (ATCC, Rockville, MD, USA) and maintained according to their recommendations. Immunofluorescence and confocal microscopy were performed as previously described [44]. Panc1 cells were plated in eight-chamber glass slides at a density of $5 \times 10^{4}$ cells/chamber and transduced with epitope-tagged (6XHis-Xpress) EZH2 $\alpha$, EZH $2 \beta$ or empty vector at MOI 150. Primary antibodies were used at the following dilutions: EZH2 $\alpha$ (1:50; described above), EZH2 $\beta$ (1:50; described above), EZH2 (1:200; Cell Signaling) and Omni-probe (D-8) (1:250; Santa Cruz). Images were obtained at $100 \times$ magnification. Frozen cryosections of human testis $(5 \mu \mathrm{m})$ were purchased from Zyagen (San Diego, CA, USA). Sections were fixed in icecold acetone for $10 \mathrm{~min}$ and rehydrated in PBS for $3 \mathrm{~min}$. Endogenous peroxidase activity was quenched using a $3 \%$ hydrogen peroxide in methanol for $20 \mathrm{~min}$ (Sigma). Avidin/Biotin blocking was performed using a kit from Vector Laboratories (Burlingame, CA, USA). Tissues were blocked in CAS Block for $1 \mathrm{~h}$ (Invitrogen) prior to overnight incubation at $4^{\circ} \mathrm{C}$ in primary antibody. Dilutions were as follows: EZH2 $\beta$ (1:200; described above) and EZH2 (1:200; Cell Signaling). Sections were subsequently washed in PBS and incubated in biotinylated goat anti-rabbit secondary antibody (Vector Laboratories) for $30 \mathrm{~min}$. Samples were incubated in Alexa Fluor-488-streptaviding conjugate (Invitrogen). Sections were counterstained with Hoescht. Images were obtained at $10 \times$ magnification.

\section{Microarray, validation and subnetwork constructions}

BxPC3 epithelial cells were plated at a density of $1 \times 10^{6}$ cells/100 $\mathrm{mm}$ dish and transduced with empty vector, EZH $2 \alpha$ or EZH2 $\beta$ (Ad5CMV) at an MOI of 150. RNA was prepared as previously described $48 \mathrm{~h}$ after transduction [44]. Experiments were performed from pooled biological triplicates in technical duplicates. The transduction efficiency of these cells at MOI 150 is $81.3 \pm$ $1.99 \%$ as determined by transduction with GFP adenovirus. Global gene expression profiling was carried out at the Microarrays Facility of the Research Center of Laval University CRCHUL using the Affymetrix Human Gene 1.0 ST arrays (28,869 well-annotated genes and 764,885 distinct probes). Intensity files were generated by Affymetrix GCS $30007 \mathrm{G}$ and the GeneChip Operating Software (Affymetrix, Santa Clara, CA, USA). Data analysis, background subtraction and intensity normalization was performed using robust multiarray analysis [45]. Genes that were differentially expressed along with false discovery rate were estimated from test $(>0.005)$ and corrected using Bayes approach $[46,47]$. Data analysis, hierarchical clustering and ontology were performed with the OneChanelGUI to extend affylmGUI graphical interface capabilities [48] and Partek Genomics Suite, version 6.5 (Partek Inc., St. Louis, MO, USA) with analysis of variance analysis. A cutoff of expression $\log _{2}$ fold change of two and $P<0.05$ was set to identify molecules whose expression was significantly differentially regulated. EZH2 $\beta$ and $\mathrm{EZH} 2 \alpha$ baseline transcript levels were assessed compared to overexpression by qPCR to assure that each isoform was expressed at approximately equivalent levels (Additional file 4: Figure S3A). Additionally, a small subset of targets was validated by qPCR (Additional file 4: Figure S3C).

Selected probes and their fold changes were loaded into IPA Software (Ingenuity Systems. Each identifier was mapped to its corresponding object in the Ingenuity Knowledge Base. These molecules, called Network Eligible molecules, were overlaid onto a global molecular network developed from information contained in the Ingenuity Knowledge Base. For the purposes of network reconstruction, a $\log _{2}$ fold change of two was used. Networks of Network Eligible molecules were then algorithmically generated based on their connectivity. The functional analysis of a network identified the biological functions and/or diseases that were most significant to the molecules in the network. The network molecules associated with biological functions and/or diseases in the Ingenuity Knowledge Base were considered for the analysis. Right-tailed Fisher's exact test was used to calculate a $P$-value determining the probability that each biological function and/or disease assigned to that network was due to chance alone.

\section{Flp-in system, transfection and luciferase assays}

The human FOXP3 core promoter containing -511 bp from transcription start site was amplified by PCR using FOXP3 promoter sequence-specific primers from position -511 to +176 . The genomic DNA extracted from $\mathrm{CD}^{+} \mathrm{T}$ cells of a healthy donor was used as a template. 
The PCR product was subcloned in the pGL3 basic vector (Promega, Madison, WI, USA). Similarly, the FOXP3 core promoter plus the first enhancer (E1) containing -511 bp to $+2,738$ was also amplified by PCR and subcloned in the pGL3 basic vector (Promega). The Flp-In system (Invitrogen) was used for the generation of a stable human FOXP3 core promoter and FOXP core $+\mathrm{E} 1$ promoter Flp-In-Jurkat. Flp-In-Jurkat cells (Invitrogen) were co-transfected with FOXP3 core or FOXP3 core + E1 in a pcDNA5/ FLP recombination target (FRT) vector and a FLP-recombinase vector (pOG44) (pOG44:FOXP3 core or FOXP3 core + E1/pcDNA5/FRT ratio $=9: 1)$, resulting in a stable integration of the gene of interest at the FRT-site in the genome. For the selective growth test, individual cells were grown in 24-well plates. The culture medium was supplemented with hygromycin at $250 \mu \mathrm{g} / \mathrm{ml}$ or $100 \mu \mathrm{g} / \mathrm{ml}$. Two million FOXP3 core and FOXP3 core + E1 Flp Jurkat cells were transfected using the Amaxa Cell Line Nucleofector Kit V for Jurkat cells according to the optimized protocol provided with the kit. Two micrograms of plasmid DNA for EZH $2 \alpha, E Z H 2 \beta$, SUZ12 and EED were used in the nucleofection procedure. Luciferase assays were done following the manufacturer's recommendations (Promega). Data represent the mean and SD of three independent experiments $\left({ }^{*} P<0.05\right)$.

\section{Adenoviral transduction and flow cytometry}

The CAR transgenic mouse was obtained through the NIAID Exchange Program, NIH: Balb/cJ[Tg]CARdelta1[Tg]DO11.10 mouse line \#4285 [49,50]. Murine naïve CD4+ splenocytes were isolated using a combination of magnetic separation kits (Miltenyi Biotec, Auburn, CA, USA). Sequential use of the CD4+CD25+ regulatory $\mathrm{T}$ cell isolation kit and the CD4+CD62L+ T cell isolation kit resulted in naïve FOXP3-negative $\mathrm{T}$ cells used for in vitro induction of FOXP3. Naïve $\mathrm{T}$ cells were isolated from the CAR transgenic Balb/cJ[Tg]CARdelta1-[Tg] DO11. Cells were activated for $48 \mathrm{~h}$ with empty vector, $\mathrm{EZH} 2 \alpha$ or EZH2 $\beta$ at an MOI of 250 . The transduction efficiency of these cells as determined by flow cytometry with propidium iodide exclusion using GFP adenovirus is $89.4 \pm 2.1 \%$. Cells were activated under the typical stimulation conditions for 3 days and processed for ChIP and qPCR to determine methylation of H3K27me3 marks at the FOXP3 core promoter and levels of FOXP3 expression, respectively. Flow cytometry was used to look at levels of FOXP3 expression within the CD4+ population across four biological replicates. Intracellular staining procedures for FOXP3 were followed using the application notes from Alexa Fluor 488 anti-mouse/rat/ human FOXP3 (BioLegend, San Diego, CA, USA). For qPCR analysis, biological triplicates were pooled and analyses performed in technical duplicate. Data represent the mean and SD of four independent experiments $\left({ }^{*} P<0.05\right)$.

\section{T cell stimulation}

In vitro activation of the isolated $\mathrm{T}$ cells followed similar conditions among the different cell types. Anti-CD3, OKT3 (eBioscience, San Diego, CA, USA) for the Jurkat cells, 145-2C11 (BD Biosciences, San Jose, CA, USA) for the mouse $\mathrm{T}$ cells, and UCHT1 (BD Biosciences) for the human $\mathrm{T}$ cells was platebound at $2 \mu \mathrm{g} / \mathrm{ml}$. Soluble anti-CD28 (BD Biosciences) at $2 \mu \mathrm{g} / \mathrm{ml}$ plus 100 units $/ \mathrm{ml}$ IL-2 was added to the cultures throughout the incubation period. Human transforming growth factor beta-1 recombinant (AUSTRAL, San Romano, CA, USA) at a concentration of $5 \mathrm{ng} / \mathrm{ml}$ was used to generate adaptive Treg cells.

\section{Chromatin immunoprecipitation assays}

ChIP assays were performed as previously described using H3-27me3 (Cell Signaling) and Omni-probe (D-8) (Santa Cruz) antibodies [51]. Primers used to analyze the FOXP3 promoter are listed in Additional file 3: Table S1. For the Polycomb target screen, mRNA and ChIP samples were processed from BxPC3 epithelial cells as described above and used with the Human Polycomb and Trithorax Target Genes ChIP PCR Array (SA Biosciences, Valencia, CA, USA). ChIP were performed in biological duplicate and of the 84 targets present on the array, $78.6 \%$ (66 out of 84 ) were occupied by EZH2 $\alpha$, serving as an internal positive experimental control. Expression profiling was performed in biological triplicate with the averaged values reported.

\section{${ }^{3} \mathrm{H}$-thymidine incorporation proliferation assay}

Naïve T cells from a CAR D011.10 mouse were isolated and transduced with empty vector, EZH $2 \alpha$ and EZH $2 \beta$ as described above. Cells were plated at $6.6 \times 10^{5} / \mathrm{ml}$ in complete Roswell Park Memorial Institute medium containing $\alpha \mathrm{CD} 28$ at $2 \mu \mathrm{g} / \mathrm{ml}$ plus 100 units/ml IL-2, and $200 \mu \mathrm{l}$ was added per well to a 96-well round bottom plate coated with $\alpha \mathrm{CD} 3$ at a concentration of $2 \mu \mathrm{g} / \mathrm{ml}$. Five days after plating, $20 \mu \mathrm{l}$ of ${ }^{3} \mathrm{H}$-thymidine (6.7 Ci/mmol NET-027) at a 1:20 dilution in complete Roswell Park Memorial Institute medium $(1.0 \mu \mathrm{Ci})$ was added to each well and incubated for approximately $18 \mathrm{~h}$. Cells were harvested and counted on the microtiter plate counter.

\section{Bioinformatics and statistical analysis}

Bioinformatics-assisted splice-mapping of the human $E Z H 2$ locus was performed using AceView [52]. An evolutionary dendrogram of common invertebrate and vertebrate EZH2 isoforms was created using the Geneious Tree Builder with a BLOSUM62 matrix, free end global alignment with a gap 
open penalty of 12 and a gap extension penalty of 3 (no outbound group selected). Predicted EZH2 splice variant sequences were curated from National Center for Biotechnology Information. Statistical analyses were performed using Graphpad Prism (La Jolla, CA, USA). Descriptive analyses including means and SDs were performed in normally distributed data. One-way analysis of variance with Tukey's post-hoc test was utilized to determine statistically significant observations. A $P$-value of $<0.05$ was considered as statistically significant.

\section{Additional files}

Additional file 1: Figure S1. Identification of multiple EZH2-positive bands in pancreatic epithelial cells. Thirty micrograms of whole cell extracts from a subset of pancreatic cells lines were examined for $\mathrm{EZH} 2 \mathrm{a}$ and $E H 2 \beta$ expression and probed with whole sera of EZH2a (1:200), $E Z H 2 \beta(1: 200)$ and EZH2aß $(1: 200)$. Note that while in some instances, EZH2 $\alpha$ and EZH2 $\beta$ are equally spliced, in other cases, only one isoform predominates. $\beta$-actin is used here as a loading control. Red arrows indicate bands of interest.

Additional file 2: Figure S2. FACS analysis of FOXP3+ cells under $\mathrm{EZH} 2 \beta$ and $\mathrm{EZH} 2 \mathrm{a}$ overexpression. Representative figure of raw data. Primary naïve murine $\mathrm{CD} 4+$ lymphocytes transduced with $E Z \mathrm{H} 2 \beta$ did not express FOXP3 upon stimulation when compared to cells transduced with empty vector. Bracket indicates the population of FOXP3+ lymphocytes from the total population of viable naive lymphocytes. Quantification of results reported in Figure $4 B$ represents the average of four biological replicates.

Additional file 3: Table S1. PCR primers. Tables of primers utilized for experiments described in this manuscript.

Additional file 4: Figure S3. Affymetrix microarray validation. (A) $q P C R$ of $E Z H 2 \beta$ and $E Z H 2 a$ expression in transduced BxPC3 epithelial cells was used to assess the levels of $\mathrm{EZH} 2 \beta$ and $\mathrm{EZH} 2 \mathrm{a}$ transcript at baseline (empty vector control) and overexpression conditions (MOI 150). Hypoxanthine phosphoribosyltransferase was used as a housekeeping control for normalization. (B) Western blot of whole cells extracts from the overexpression conditions described in A probed with antibodies against EZH2a, EZH2 $\beta$ and HIS-tag. $\beta$-actin was used as a loading control. (C) To validate the results of the Affymetrix GeneChip Human Gene 1.0 ST microarray, five targets were selected for validation via qPCR. Results are presented as a scaled, comparative heatmap.

\section{Abbreviations}

BLAST: Basic Local Alignment Search Tool; bp: Base pair; ChIP: Chromatin immunoprecipitation; CMV: Cytomegalovirus; EED: Embryonic ectoderm development; EZH2: Enhancer of zeste homologue 2; GFP: Green fluorescent protein; H3-K27me3: Trimethylation of histone 3 at lysine 27; HIS: Histidine; HMT: Histone methyltransferase; Ig: Immunoglobulin; IL: Interleukin; IPA: Ingenuity Pathways Analysis; kDa: kiloDalton; MOI: Multiplicity of infection; PBS: Phosphate-buffered saline; PCR: Polymerase chain reaction; PRC: Polycomb repressive complex; qPCR: Quantitative polymerase chain reaction; RBBP: Retinoblastoma binding protein; RT-PCR: Reverse transcription polymerase chain reaction; SD: Standard deviation; SUZ12: Suppressor of zeste 12; TBST: Tris buffered saline with Tween.

\section{Competing interests}

The authors declare that they have no competing interests.

\section{Authors' contributions}

$A G, G L$ and $R U$ generated the main idea of the work and developed the study design, both conceptually and methodologically. AG, PS, AM, EC and YX made substantial contributions to acquisition of data. AG, GL, PS, AM, YX, $E C, J, W F$ and $R U$ contributed to analysis and interpretation of data. AG, GL, $P S, A M, Y X, E C, J l, W F$ and $R U$ were in charge of writing the manuscript from first draft to completion. AG, GL, PS, AM, YX, EC, Jl, WF and RU made comments, suggested appropriate modifications and corrections that were included in the final version of this article, which all authors read and approved.

\section{Acknowledgements}

This work was supported by funding from National Institutes of Health grants DK52913 (RU), Fraternal Order of Eagles Cancer Award (GL), T32CA148073 (AG), the Mayo Clinic Center for Cell Signaling in Gastroenterology (P30DK084567), and the Mayo Foundation.

\section{Author details}

'Laboratory of Epigenetics and Chromatin Dynamics, Mayo Clinic, Rochester, MN 55905, USA. "2Molecular Endocrinology and Oncology Research Center, CHUL Research Center, Quebec, Canada. ${ }^{3}$ INSERM U.624, Stress Cellulaire, 163 Avenue de Luminy, Case 915, Parc Scientifique et Technologique de Luminy, 13288, Marseille Cedex 9, France. ${ }^{4}$ Translational Epigenomics Program, Center for Individualized Medicine (CIM), Mayo Clinic, Rochester, MN 55905, USA. ${ }^{5}$ Departments of Medicine, Physiology and Biochemistry, Mayo Clinic, Rochester, MN 55905, USA.

Received: 25 April 2012 Accepted: 5 February 2013

Published: 28 February 2013

\section{References}

1. Margueron R, Reinberg D: The Polycomb complex PRC2 and its mark in life. Nature 2011, 469:343-349.

2. Tavares L, Dimitrova E, Oxley D, Webster J, Poot R, Demmers J, Bezstarosti K, Taylor S, Ura H, Koide H, Wutz A, Vidal M, Elderkin S, Brockdorff N: RYBPPRC1 complexes mediate H2A ubiquitylation at polycomb target sites independently of PRC2 and H3K27me3. Cell 2012, 148:664-678.

3. Cao R, Wang L, Wang H, Xia L, Erdjument-Bromage H, Tempst P, Jones RS, Zhang Y: Role of histone H3 lysine 27 methylation in Polycomb-group silencing. Science 2002, 298:1039-1043.

4. Czermin B, Melfi R, McCabe D, Seitz V, Imhof A, Pirrotta V: Drosophila enhancer of Zeste/ESC complexes have a histone $\mathrm{H} 3$ methyltransferase activity that marks chromosomal Polycomb sites. Cell 2002, 111:185-196.

5. Cao R, Zhang Y: SUZ12 is required for both the histone methyltransferase activity and the silencing function of the EED-EZH2 complex. Mol Cell 2004, 15:57-67.

6. Denisenko O, Shnyreva M, Suzuki H, Bomsztyk K: Point mutations in the WD40 domain of Eed block its interaction with Ezh2. Mol Cell Biol 1998, 18:5634-5642

7. Han Z, Xing X, Hu M, Zhang Y, Liu P, Chai J: Structural basis of EZH2 recognition by EED. Structure 2007, 15:1306-1315.

8. Margueron R, Justin N, Ohno K, Sharpe ML, Son J, Drury WJ 3rd, Voigt P, Martin SR, Taylor WR, De Marco V, Pirrotta V, Reinberg D, Gamblin SJ: Role of the polycomb protein EED in the propagation of repressive histone marks. Nature 2009, 461:762-767.

9. Tie F, Stratton CA, Kurzhals RL, Harte PJ: The N terminus of Drosophila ESC binds directly to histone $\mathrm{H} 3$ and is required for $\mathrm{E}(\mathrm{Z})$-dependent trimethylation of H3 lysine 27. Mol Cell Biol 2007, 27:2014-2026.

10. Margueron R, Li G, Sarma K, Blais A, Zavadil J, Woodcock CL, Dynlacht BD, Reinberg D: Ezh1 and Ezh2 maintain repressive chromatin through different mechanisms. Mol Cell 2008, 32:503-518.

11. Tie F, Furuyama T, Prasad-Sinha J, Jane E, Harte PJ: The Drosophila Polycomb group proteins ESC and E(Z) are present in a complex containing the histone-binding protein $\mathrm{p} 55$ and the histone deacetylase RPD3. Development 2001, 128:275-286.

12. Sarma K, Margueron R, Ivanov A, Pirrotta V, Reinberg D: Ezh2 requires PHF1 to efficiently catalyze $\mathrm{H} 3$ lysine 27 trimethylation in vivo. Mol Cell Biol 2008, 28:2718-2731.

13. Schuettengruber B, Chourrout D, Vervoort M, Leblanc B, Cavalli G: Genome regulation by polycomb and trithorax proteins. Cell 2007, 128:735-745

14. Kuzmichev A, Jenuwein T, Tempst P, Reinberg D: Different EZH2containing complexes target methylation of histone $\mathrm{H} 1$ or nucleosomal histone H3. Mol Cell 2004, 14:183-193.

15. Kuzmichev A, Margueron R, Vaquero A, Preissner TS, Scher M, Kirmizis A, Ouyang X, Brockdorff N, Abate-Shen C, Farnham P, Reinberg D: Composition and histone substrates of polycomb repressive group 
complexes change during cellular differentiation. Proc Natl Acad Sci U S A 2005, 102:1859-1864.

16. Sparmann A, van Lohuizen M: Polycomb silencers control cell fate, development and cancer. Nat Rev Cancer 2006, 6:846-856.

17. Kleer CG, Cao Q, Varambally S, Shen R, Ota I, Tomlins SA, Ghosh D, Sewalt RG, Otte AP, Hayes DF, Sabel MS, Livant D, Weiss SJ, Rubin MA, Chinnaiyan AM: $\mathrm{EZH} 2$ is a marker of aggressive breast cancer and promotes neoplastic transformation of breast epithelial cells. Proc Natl Acad Sci U S A 2003, 100:11606-11611.

18. Varambally S, Dhanasekaran SM, Zhou M, Barrette TR, Kumar-Sinha C, Sanda MG, Ghosh D, Pienta KJ, Sewalt RG, Otte AP, Rubin MA, Chinnaiyan AM: The polycomb group protein $\mathrm{EZH} 2$ is involved in progression of prostate cancer. Nature 2002, 419:624-629.

19. Bohrer LR, Chen $\mathrm{S}$, Hallstrom TC, Huang $H$ : Androgens suppress EZH2 expression via retinoblastoma (RB) and p130-dependent pathways: a potential mechanism of androgen-refractory progression of prostate cancer. Endocrinology 2010, 151:5136-5145.

20. Bracken AP, Pasini D, Capra M, Prosperini E, Colli E, Helin K: EZH2 is downstream of the pRB-E2F pathway, essential for proliferation and amplified in cancer. EMBO J 2003, 22:5323-5335.

21. Kotake $Y$, Cao R, Viatour $P$, Sage J, Zhang Y, Xiong Y: pRB family proteins are required for $\mathrm{H} 3 \mathrm{~K} 27$ trimethylation and Polycomb repression complexes binding to and silencing p16INK4alpha tumor suppressor gene. Genes Dev 2007, 21:49-54.

22. Shi B, Liang J, Yang $X$, Wang $Y$, Zhao $Y$, Wu H, Sun L, Zhang $Y$, Chen $Y$, Li R, Zhang $Y$, Hong $M$, Shang $Y$ : Integration of estrogen and Wnt signaling circuits by the polycomb group protein EZH2 in breast cancer cells. Mol Cell Biol 2007, 27:5105-5119.

23. Tang X, Milyavsky M, Shats I, Erez N, Goldfinger N, Rotter V: Activated p53 suppresses the histone methyltransferase EZH2 gene. Oncogene 2004, 23:5759-5769.

24. Tonini T, Bagella L, D'Andrilli G, Claudio PP, Giordano A: Ezh2 reduces the ability of HDAC1-dependent pRb2/p130 transcriptional repression of cyclin A. Oncogene 2004, 23:4930-4937.

25. Bryant RJ, Cross NA, Eaton CL, Hamdy FC, Cunliffe VT: EZH2 promotes proliferation and invasiveness of prostate cancer cells. Prostate 2007, 67:547-556

26. Cao Q, Yu J, Dhanasekaran SM, Kim JH, Mani RS, Tomlins SA, Mehra R, Laxman B, Cao X, Kleer CG, Varambally S, Chinnaiyan AM: Repression of $\mathrm{E}$-cadherin by the polycomb group protein $\mathrm{EZH} 2$ in cancer. Oncogene 2008, 27:7274-7284.

27. Fujii S, Ochiai A: Enhancer of zeste homolog 2 downregulates E-cadherin by mediating histone $\mathrm{H} 3$ methylation in gastric cancer cells. Cancer Sci 2008, 99:738-746.

28. Lu C, Han HD, Mangala LS, Ali-Fehmi R, Newton CS, Ozbun L, Armaiz-Pena GN, Hu W, Stone RL, Munkarah A, Ravoori MK, Shahzad MM, Lee JW, Mora E, Langley RR, Carroll AR, Matsuo K, Spannuth WA, Schmandt R, Jennings NB, Goodman BW, Jaffe RB, Nick AM, Kim HS, Guven EO, Chen YH, Li LY, Hsu MC, Coleman RL, Calin GA, et al: Regulation of tumor angiogenesis by EZH2. Cancer Cell 2010, 18:185-197.

29. Zeidler M, Kleer CG: The Polycomb group protein enhancer of zeste 2: its links to DNA repair and breast cancer. J Mol Histol 2006, 37:219-223.

30. Zeidler M, Varambally S, Cao Q, Chinnaiyan AM, Ferguson DO, Merajver SD, Kleer CG: The Polycomb group protein EZH2 impairs DNA repair in breast epithelial cells. Neoplasia 2005, 7:1011-1019.

31. Nolz JC, Gomez TS, Billadeau DD: The Ezh2 methyltransferase complex: actin up in the cytosol. Trends Cell Biol 2005, 15:514-517.

32. Yamamoto K, Sonoda M, Inokuchi J, Shirasawa S, Sasazuki T: Polycomb group suppressor of zeste 12 links heterochromatin protein 1alpha and enhancer of zeste 2. J Biol Chem 2004, 279:401-406.

33. Bracken AP, Dietrich N, Pasini D, Hansen KH, Helin K: Genome-wide mapping of Polycomb target genes unravels their roles in cell fate transitions. Genes Dev 2006, 20:1123-1136.

34. Xiong Y, Khanna S, Grzenda AL, Sarmento OF, Svingen PA, Lomberk GA, Urrutia RA, Faubion WA Jr: Polycomb antagonizes p300/CREB-binding protein-associated factor to silence FOXP3 in a Kruppel-like factor-dependent manner. J Biol Chem 2012, 287:34372-34385.

35. Ku M, Koche RP, Rheinbay E, Mendenhall EM, Endoh M, Mikkelsen TS, Presser A, Nusbaum C, Xie X, Chi AS, Adli M, Kasif S, Ptaszek LM, Cowan CA, Lander ES, Koseki H, Bernstein BE: Genomewide analysis of PRC1 and
PRC2 occupancy identifies two classes of bivalent domains. PLOS Genet 2008, 4:e1000242.

36. Cardoso C, Mignon C, Hetet G, Grandchamps B, Fontes M, Colleaux L: The human $\mathrm{EZH} 2$ gene: genomic organisation and revised mapping in $7 \mathrm{q} 35$ within the critical region for malignant myeloid disorders. Eur J Hum Genet 2000, 8:174-180.

37. Hobert $O$, Jallal $B$, Ullrich A: Interaction of Vav with ENX-1, a putative transcriptional regulator of homeobox gene expression. Mol Cell Biol 1996, 16:3066-3073.

38. Shen X, Liu Y, Hsu YJ, Fujiwara Y, Kim J, Mao X, Yuan GC, Orkin SH: EZH1 mediates methylation on histone $\mathrm{H} 3$ lysine 27 and complements $\mathrm{EZH} 2$ in maintaining stem cell identity and executing pluripotency. Mol Cell 2008, 32:491-502.

39. Schotta G, Lachner M, Sarma K, Ebert A, Sengupta R, Reuter G, Reinberg D, Jenuwein $\mathrm{T}$ : A silencing pathway to induce H3-K9 and H4-K20 trimethylation at constitutive heterochromatin. Genes Dev 2004, 18:1251-1262.

40. Hyland PL, McDade SS, McCloskey R, Dickson GJ, Arthur K, McCance DJ, Patel D: Evidence for alteration of EZH2, BMI1, and KDM6A and epigenetic reprogramming in human papillomavirus type 16 E6/E7expressing keratinocytes. J Virol 2011, 85:10999-11006.

41. Su IH, Basavaraj A, Krutchinsky AN, Hobert O, Ullrich A, Chait BT, Tarakhovsky A: Ezh2 controls B cell development through histone $\mathrm{H} 3$ methylation and Igh rearrangement. Nat Immunol 2003, 4:124-131.

42. Schwartz YB, Pirrotta V: Polycomb silencing mechanisms and the management of genomic programmes. Nat Rev Genet 2007, 8:9-22.

43. Zeng $X$, Chen $S$, Huang $H$ : Phosphorylation of EZH2 by CDK1 and CDK2: a possible regulatory mechanism of transmission of the $\mathrm{H} 3 \mathrm{~K} 27 \mathrm{me} 3$ epigenetic mark through cell divisions. Cell Cycle 2011, 10:579-583.

44. Gebelein B, Urrutia R: Sequence-specific transcriptional repression by KS1, a multiple-zinc-finger-Kruppel-associated box protein. Mol Cell Biol 2001, 21:928-939.

45. Irizarry RA, Hobbs B, Collin F, Beazer-Barclay YD, Antonellis KJ, Scherf U, Speed TP: Exploration, normalization, and summaries of high density oligonucleotide array probe level data. Biostatistics 2003, 4:249-264.

46. Prasanth SG, Prasanth KV, Siddiqui K, Spector DL, Stillman B: Human Orc2 localizes to centrosomes, centromeres and heterochromatin during chromosome inheritance. EMBO J 2004, 23:2651-2663.

47. Kondo Y, Shen L, Ahmed S, Boumber Y, Sekido Y, Haddad BR, Issa JP: Downregulation of histone $\mathrm{H} 3$ lysine 9 methyltransferase G9a induces centrosome disruption and chromosome instability in cancer cells. PLOS One 2008, 3:e2037.

48. Wettenhall JM, Simpson KM, Satterley K, Smyth GK: AffylmGUI: a graphical user interface for linear modeling of single channel microarray data. Bioinformatics 2006, 22:897-899.

49. Murphy KM, Heimberger AB, Loh DY: Induction by antigen of intrathymic apoptosis of CD4+CD8+TCRlo thymocytes in vivo. Science 1990, 250:1720-1723.

50. Bao Y, Peng W, Verbitsky A, Chen J, Wu L, Rauen KA, Sawicki JA: Human coxsackie adenovirus receptor (CAR) expression in transgenic mouse prostate tumors enhances adenoviral delivery of genes. Prostate 2005 64:401-407.

51. Lomberk G, Mathison AJ, Grzenda A, Seo S, Demars CJ, Rizvi S, Bonilla-Velez J, Calvo E, Fernandez-Zapico ME, lovanna J, Buttar NS, Urrutia R: Sequencespecific recruitment of heterochromatin protein 1 via interaction with Krüppel-like factor 11, a human transcription factor involved in tumor suppression and metabolic diseases. J Biol Chem 2012, 287:13026-13039.

52. Thierry-Mieg D, Thierry-Mieg J: AceView: a comprehensive cDNAsupported gene and transcripts annotation. Genome Biol 2006, 7(Suppl 1): S12.11-14.

doi:10.1186/1756-8935-6-3

Cite this article as: Grzenda et al:: Functional characterization of EZH2 $\beta$ reveals the increased complexity of $\mathrm{EZH} 2$ isoforms involved in the regulation of mammalian gene expression. Epigenetics \& Chromatin 2013 $6: 3$. 